\title{
Comparison Between Dielectric Inversion Results from Synthetic Aperture Radar Co- and Quad-Polarimetric Data via a Polarimetric Two- Scale Model
}

\author{
Cornelius Quigley, Camilla Brekke, Member, IEEE, Torbjørn Eltoft, Member, IEEE
}

\begin{abstract}
In this study we compare the retrieval results for the dielectric properties of verified oil slick, acquired using airborne multifrequency synthetic aperture radar. A polarimetric two-scale model was used to invert the radar imagery by first employing solely the co-polarization channels, and then by employing the co-polarization channels in conjunction with the cross- polarization channels, and thereby employing the full suite of polarization information available. The goal is to show that the inversion results obtained from both methods are consistent. Given that the ocean surface is a highly nondepolarizing surface scatter, the signal return within the cross-polarization channels is usually negligible and of no practical use when trying to invert the returned backscatter into useful quantities such as the dielectric constant. In this paper, we employ F-SAR data, which was acquired in X-, Sand L-bands and has an extremely low noise floor, implying that the cross-polarization ratio can be employed. A signalto-noise analysis showed that only the L-band acquisitions were suitable for analysis in this paper. The retrieval results are comparable for the two methods in the case of low dielectric values.
\end{abstract}

Index Terms - dielectric properties, oil spill, surface slick characterization, synthetic aperture radar

\section{INTRODUCTION}

$\mathrm{M}$ ineral oils may be introduced into the marine environment via two broad mechanisms. The first being natural seeps, where unprocessed gaseous or liquid hydrocarbons may escape through fractures and sediments on the ocean floor. The second mechanism alludes to activities associated with industrial petroleum extraction and ship transport which can introduce mineral oils into the marine environment via intentional acts of pollution or ship or oil rig accidents. According to [1] approximately one-half to twothirds of all oil discharged into the ocean comes from

The NORSE2019 campaign was funded by CIRFA; CIRFA partners and the Research Council of Norway under Grant 237906.

C. Quigley, T. Eltoft and C. Brekke are with the Department of Physics and Technology, UiT - The Arctic University of Norway, NO-9037 Tromsø, Norway (e-mail: cornelius.p.quigley@uit.no, torbjorn.eltoft@uit.no, camilla.brekke@uit.no).

Manuscript received anthropogenic sources. Carpenter [2] estimates that each year, between 2000 to 3000 instances of illegal dumping of oil take place in European waters. The effects of the presence of mineral oil can result in disastrous consequences for, e.g. ecologically sensitive wetlands, coral reefs, and fishing grounds [3].

Given the consequences oil spills can have on marine wildlife, as well as the impact they can take on local economies, continuous and efficient monitoring technology is required to provide synoptic surveillance on industrial activities as well as providing timely information to direct clean-up efforts. In this regard, both airborne and spaceborne synthetic aperture radar (SAR) have proven themselves to be the most effective technologies for oil spill remote sensing, due to their insensitivity to atmospheric and lighting conditions and the high level of resolution that can be achieved. Given the high cost associated with operating aircraft, and the vast distances that are required to be monitored, airborne SAR is not a good candidate for operational purposes and is generally regarded as an experimental instrument, which is also the case in this study. A compelling example of the application of spaceborne SAR for these purposes occurred during the destruction of the Deepwater Horizon platform in the Gulf of Mexico on 20 April 2010. Throughout the incident, approximately 1400 spaceborne SAR scenes from more than 700 satellite passes were used to establish the extent and trajectory of the oil spill [3].

Mineral oils materialize within SAR imagery as dark areas in contrast to the surrounding ocean. The underlying physical mechanisms that cause this are two-fold. Firstly, surface slicks dampen short-scale capillary waves, which cause a reduction in the overall short-scale roughness of the ocean surface. A smoother ocean surface increases forward scattering which results in a reduction of backscatter to the sensor [4]. The second mechanism, is a result of the assortment of mechanisms that act upon oil when it is included into the marine environment, collectively referred to as weathering [1]. The most relevant of these processes is emulsification, whereby sea water can become entrained within the slick. This mixture of sea water and oil has the effect of altering the electrical permittivity of the scattering surface resulting in a reduction of backscatter to the SAR if the mineral oil is mixed in high enough concentrations within a thin layer below the ocean surface. A lower dielectric constant implies that less energy is reflected by the scattering surface. The capability of SAR to observe fluctuations in the dielectric properties of a marine scattering surface, due to the presence of oil-in-water mixtures, 

MONTH 20XX

is reliant on the thickness of the emulsion layer relative to the wavelength of the sensing radiation. As an example, [5] states that for L-band SAR (with wavelength of $24 \mathrm{~cm}$ ), the thickness of the scattering surface would need to be a few millimeters in scale. This sensitivity of SAR to oil slick thickness was illustrated by [6] who showed that infra-red (IR) airborne data, which is believed to be sensitive to the thickness regimes within oil slick, showed a strong correlation with SAR data, indicating that SAR has the ability to discern the thickness information within slick and hence dielectric properties.

With the use of theoretical scattering models that are dependent on key geophysical parameters, it should be possible to invert the returned backscatter to the SAR for the effective permittivity of oil-water emulsion mixtures that are present on the ocean surface. Retrieved values for the dielectric permittivity of the scattering material can then be utilized in a mixture model that relates the permittivity of pure crude oil, ocean water and the volumetric content of sea water within emulsion, in order to provide a pixel-by-pixel assessment of the oil-to-sea water ratio. This information can be used by first responders to possibly determine the amount of oil spilled, an important parameter that is evaluated in any oil spill event [7], or to provide a heuristic based assessment on where there are greater amounts of oil within slick compared to other areas, thereby allowing first responders to target areas of a slick that contain a higher concentration of oil.

Some notable studies that attempt to determine the amount of oil mixed with water from the Normalized Radar Cross Section (NRCS) or polarimetric features, include [5, 8], who employ a procedure that aims to relate the measured backscatter to the Bonn Agreement Oil Appearance Codes (BAOAC) for aerial observers of oil spills [9]. The result is an index, varying between -1 to 1 and is called the oil/water mixing index (Mdex). The purpose of the Mdex was to promote a shared communication code for optical and SAR observations.

Angelliaume et. al. [10] provided a methodology to determine the amount of oil pollutant contained within an oil emulsion via the use of the Universal Weighted Curvature Approximation (U-WCA) scattering model in L-band SAR imagery. The conclusions of the study state that by using SAR data with a high SNR, viable estimations for the volumetric content of oil within a pixel may be inferred. The authors also state that their methodology is robust to sea surface state.

This paper is a continuation of the work presented in Quigley et. al. [11]. In that study, the authors applied the Polarimetric Two-Scale Model (PTSM), that was developed by [12] for the purposes of estimating soil moisture content via the use of fully polarimetric SAR, to invert the co- polarization ratio to obtain estimates for the absolute value of the complex permittivity $|\varepsilon|$ of verified oil slick. The model in its original form was depended upon the complex quantity $\varepsilon$ and not the absolute value of $|\varepsilon|$. The PTSM is a theoretical scattering model that improves upon other well-established models such as the XBragg model which was proposed by [13]. A discussion on this point can be found in [11]. However, [11] showed that using the model with the absolute value for the electrical permittivity instead of the complex value, did not lead to significant errors in retrieval results, with errors ranging from approximately 0 to 1.2 for values of $|\varepsilon|$ ranging from 0 to 20 , with a value of $|\varepsilon|$ equal to 20 being the value at which the slick and the surrounding ocean were found to become indistinguishable. For the sake of computational simplicity $|\varepsilon|$ will be substituted into the PTSM in this study.

In [11], the authors are only able to utilize the copolarization channels, as the cross- polarization channels were redundant due to noise corruption of the C-band space borne sensor (Radarsat-2) used in that study. As the use of the copolarization ratio results in an equation with two unknowns, i.e. $s$ (a parameter related to the large-scale roughness of the scattering surface which will be explained in section II.A) and $|\varepsilon|,[11]$ employed a procedure to estimate $s$, by employing square regions, $100 \times 100$ pixels in size adjacent to the slick, to act as estimators for the values of $s$ within slick. However, if there is information in the cross- polarization channels, in the form of a high signal-to-noise ratio (SNR), a series of look-up charts can be created by plotting the co- polarization ratio against the cross- polarization ratio and simultaneous values for $s$ and $|\varepsilon|$ can be determined. The implicit assumption, is that this will yield more accurate estimations for $|\varepsilon|$ since assumptions about $s$ don't have to be made a priori. This procedure is investigated in this paper.

In this study, the PTSM was applied to quad- polarization data sets acquired by Deutsches Zentrum für Luft- und Raumfahrt (DLR) F-SAR instrument in X-, S- and L-bands acquired simultaneously during the NOrwegian Radar oil Spill Experiment in 2019 (NORSE 2019) led by the Centre for Integrated Remote Sensing and Forecasting for Arctic Operations (CIRFA) as an embedded part of the Norwegian Clean Seas Association for Operating Companies (NOFO) annual oil-on-water campaign. This campaign took place in the North Sea in June 2019. F-SAR, being an airborne instrument, has the capability to acquire data with a low level of noise corruption, resulting in data sets with a high SNR in all polarimetric channels.

The PTSM was applied to the selected data sets in two ways. Firstly, the methodology outlined in [11] where only the copolarization channels were utilized, and which is outlined in Appendix B, was employed (hereinafter referred to as method 1). Secondly, the method outlined in [11] was implemented where the cross-polarization channels are utilized in conjunction with the co- polarization channels (hereinafter referred to as method 2). This will be outlined in section II.B. As already stated, since no assumptions about $s$ need to be made when the full suite of polarimetric information is available, the retrieval results obtained from the latter method can act as a reference for the former method employed. The results indicate that for low dielectric values (below 8 approximately), retrieved $|\varepsilon|$ values are consistent across both methods.

This paper is organized as follows. Section II will introduce the PTSM as well as the two inversion procedures for $|\varepsilon|$ that are reliant on either the co-polarization ratio or the copolarization ratio and cross- polarization ratio. Section III will introduce the data set as well as a noise analysis that was undertaken to assess the viability of using the full set of polarimetric information provided by the F-SAR instrument. Section IV introduces a sensitivity analysis of the PTSM model. Section V shows the results of the inversion procedures. Section 

MONTH 20XX

VI will attempt to link the results obtained for $|\varepsilon|$ to photographs of the discharged mineral oil slick. Section VII concludes the paper.

\section{PTSM AND INVERSION PROCEDURES}

The following subsections will provide a brief overview of the PTSM, as well as outlining the methodology in which it is inverted in order to determine values for $|\varepsilon|$, i.e. method 1 and method 2. As will be highlighted in Section III.C, the crosspolarization ratio is only viable for use when there is a high signal-to-noise ratio in the cross-polarization channels. It should be noted that the inversion procedure that was implemented using only the co-polarization channels (method 1) was developed by [11] and is outlined in that paper. For ease of reference for the reader that same procedure is reproduced in Appendix B.

\section{A. PTSM}

The PTSM was derived by [12]. The model considers the ocean surface to be a collection of randomly assorted tilted facets. The facet slopes, along the range and azimuth directions, $a$ and $b$, are assumed to be identically distributed zero-mean $s^{2}$ variance Gaussian random variables, i.e. $a, b \sim N\left(0, s^{2}\right)$. Under the condition of no facet tilt, the Small Perturbation Model (SPM) is assumed to be valid. The parameter $s$ here is the largescale roughness indicator.

Given the models dependence on the small-scale roughness parameter, $h$ (see Appendix A), the polarization ratios are employed. This insures that the small-scale roughness spectrum of the scattering surface cancels out, and the resulting ratios only depend on three primary unknown parameters, $|\varepsilon|, s$ and, $H_{t}$, the Hurst coefficient, a quantity that is related to the fractal dimension of the scattering surface (see Appendix A for a more thorough treatment). It should be noted that the resulting ratios also depend on the incidence angle of the radiation which can be obtained from the SAR data).

The polarization ratios are defined as

$$
\left\{\begin{array}{c}
C o-p o l=\frac{\left\langle\sigma_{V V}^{0}\right\rangle / a, b}{\left\langle\sigma_{H H}^{0}\right\rangle_{/ a, b}} \\
\text { Cross }-p o l=\frac{\left\langle\sigma_{H V}^{0}\right\rangle / a, b}{\left\langle\sigma_{V V}^{0}\right\rangle / a, b}
\end{array}\right.
$$

The notation $\langle\ldots\rangle_{/ a, b}$ refers to ensemble averaging with respect to the facet slopes $a$ and $b$, indicating that the return from within a single resolution cell is averaged over the facet slopes $a$ and $b$ from multiple facets. Note, the VV NRCS channel was chosen as the denominator in the crosspolarization channel for the sake of this study as the return in the $\mathrm{VV}$ channel is greater than the $\mathrm{HH}$ channel over ocean areas. The choice of the HV channel is arbitrary as it is generally accepted that it is numerically similar to the $\mathrm{VH}$ channel for backscatter from natural media.

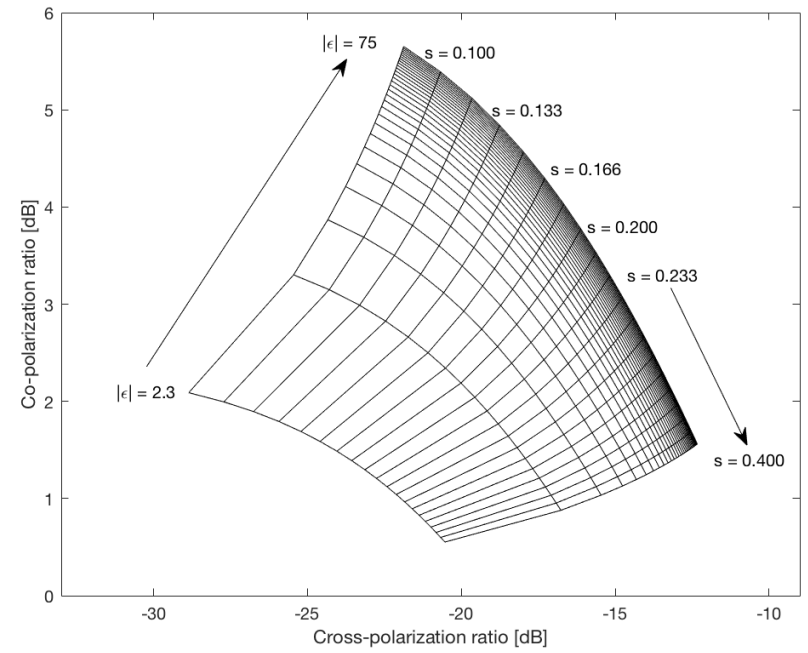

Fig.1 PTSM plot of co-polarization ratio against crosspolarization ratio for angle of incidence, $v=40^{\circ}$, radiation frequency of $5.4 \mathrm{GHz}$, and Hurst coefficient $H_{t}=0.9$. The reader is referred to Appendix A for a discussion on this parameter.

\section{B. Estimating $|\varepsilon|$ utilizing co- and cross- polarization ratios (Method 2)}

When utilizing the co- and cross-polarization ratios for the estimation of $|\varepsilon|$, we are left with two equations that are dependent on $|\varepsilon|$ and $s$. This provides the opportunity to create a series of look-up charts, evaluated for each incidence angle value, $v$, across the SAR image in range direction, by plotting the PTSM co- and cross-polarization ratios against each other.

Fig. 1 shows the PTSM polarization ratios plotted for a frequency of radiation equal to $5.4 \mathrm{GHz}$, a look angle of $40^{\circ}$ and $H_{t}$ equal to 0.9 . By simultaneously plotting the scatter of coand cross-polarization values taken from the SAR imagery, simultaneous values for $|\varepsilon|$ and $s$ can be determined.

\section{EXPERIMENTAL SETUP AND DATA ACQUISITION}

The following subsections provide a brief overview of the technical aspects of the F-SAR instrument, followed by a brief outline of the NORSE 2019 oil-on-water campaign. It should be noted that this section is designed to illustrate the information relevant to this study with a more comprehensive account of the NORSE 2019 oil-on-water experiment in the process of being published [14].

\section{A. $F-S A R$}

The F-SAR platform is a Dornier DO228-212 aircraft mounted with X-, C-, S-, L- and P-band polarimetric SAR that operates with bandwidths of 760, 400, 300, 150 and 100/50 $\mathrm{MHz}$, respectively. The total frequency range of the instrument is $0.35-9.6 \mathrm{GHz}$ [15]. The instrument is capable of transmitting and receiving in both horizontal $(\mathrm{H})$ and vertical (V) configurations resulting in imagery in $\mathrm{HH}, \mathrm{HV}, \mathrm{VV}$ and $\mathrm{VH}$ polarization channels, and is capable of acquiring simultaneous imagery in different frequency bands. For regular Earth observation purposes, the radar is capable of acquiring imagery at off-nadir look angles ranging between $25^{\circ}$ to $60^{\circ}$ at altitudes 

MONTH 20XX

up to $6000 \mathrm{~m}$ above sea level, the maximum operating altitude of the DO228 aircraft [15].

\section{B. NORSE 2019}

NOFO conducts annual oil-on-water exercises for the purposes of verifying clean-up procedures and testing new equipment. In 2019, the oil-on-water campaign was conducted in the North Sea (N $\left.59^{\circ} 59^{\prime}, \mathrm{E} 2^{\circ} 27^{\prime}\right)$. In this paper, we test the PTSM on data acquired during the NORSE 2019 experiment, led by UiT and MET Norway, that took place as an integrated part of NOFO's 2019 campaign.

During the exercise, two flights with the F-SAR mounted Dornier DO228-212 aircraft were conducted, acquiring imagery in X-, S- and L-bands, on 12 June 2019. Flight 1 was conducted between 05:12 - 06:33 UTC and acquired 12 acquisitions while flight 2 was conducted between 10:50 12:17 UTC and acquired 14 acquisitions. Fight 1 acquired data while the mineral oil emulsion and soybean oil were being discharged at times of 05:30 - 05:54 UTC and 06:20 - 06:37 UTC, respectively. As a result, the mineral oil emulsion and soybean oil are confined to a small area on the ocean surface. As a consequence, scenes from flight 1 were not considered for analysis in this study.

Two scenes, out of the 14 that were acquired in flight 2, were chosen for this study based on appropriate noise characteristics of the imagery, which will be discussed in section III.C, and where the head of the mineral oil slick, i.e. the portion of the slick where there was a greater concentration of oil, had incidence angles greater than $35^{\circ}$. Incidence angles less than $35^{\circ}$ approximately are not applicable, as the sensitivity of the radar to small-scale roughness undulations is low under this threshold, which violates the small perturbation assumption that is used to derive the PTSM $[4,16]$.

Fig. 2 shows the two L-band F-SAR acquisitions of the verified slick of mineral oil emulsion that was discharged during the campaign. In total $2 \mathrm{~m}^{3}$ of mineral oil emulsion was discharged onto the ocean surface. The discharged emulsion was composed of $45 \%$ oil (10 parts evaporated Oseberg Blend and 1 part IF0 380) and 55\% sea water. The viscosity was between $1500-1600 \mathrm{mPa} \cdot \mathrm{s}$. IFO 380 refers to Intermediate Fuel Oil with viscosity $\leq 380 \mathrm{cSt}(<3.5 \%$ Sulphur $)$.

Table 1: Properties of SAR acquisitions and in-situ information

\begin{tabular}{|c|c|c|}
\hline Scene ID & A1 & A2 \\
\hline Date & 12 June 2019 & 12 June 2019 \\
\hline Time (UTC) & 11:03 & $11: 49$ \\
\hline $\begin{array}{l}\text { Incidence angle (at } \\
\text { emulsion slick) }\end{array}$ & $51.2^{\circ}-56.9^{\circ}$ & $44.5^{\circ}-52.0^{\circ}$ \\
\hline Sensor velocity $[\mathrm{m} / \mathrm{s}]$ & 90.6 & 89.8 \\
\hline Sensor altitude [m] & 2497.4 & 2498.8 \\
\hline $\begin{array}{l}\text { Age of emulsion at time } \\
\text { of acquisition [hours] }\end{array}$ & $\sim 5-5.5$ & $\sim 6-6.3$ \\
\hline $\begin{array}{l}\text { Resolution }(\mathrm{Rg} \times \mathrm{Az}) \\
{[\mathrm{m}]_{(\mathrm{X}-\mathrm{S}-\mathrm{S}, \mathrm{L}-\mathrm{Band})}}\end{array}$ & \multicolumn{2}{|c|}{$(0.2 \times 0.2),(0.5 \times 0.35),(1 \times 0.4)^{*}$} \\
\hline $\begin{array}{l}\text { Pixel spacing }(\mathrm{Rg} \times \mathrm{Az}) \\
{[\mathrm{m}]_{(\mathrm{X}-, \mathrm{S}-, \mathrm{L}-\mathrm{Band})}}\end{array}$ & \multicolumn{2}{|c|}{$(0.60 \times 0.18),(0.60 \times 0.18),(0.60 \times 0.36)$} \\
\hline $\begin{array}{l}\text { Frequency of radiation } \\
{[\mathrm{GHz}]_{(\mathrm{X}-\mathrm{S}-\mathrm{S}-\mathrm{L}-\mathrm{Band})}}\end{array}$ & \multicolumn{2}{|c|}{$9.6,3.25,1.325$} \\
\hline Wind speed $[\mathrm{m} / \mathrm{s}]$ & \multicolumn{2}{|c|}{12} \\
\hline
\end{tabular}

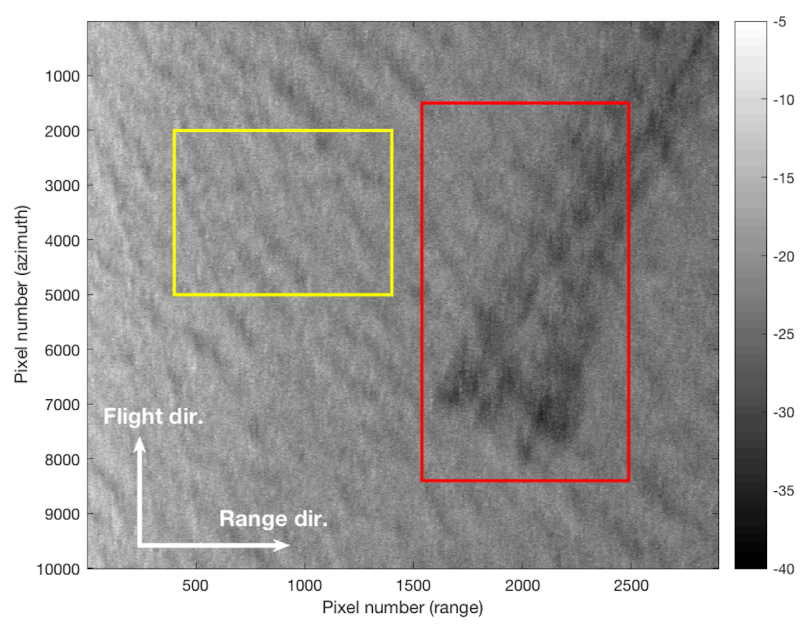

(a) L-band F-SAR scene 12 June 2019, 11:03 UTC.

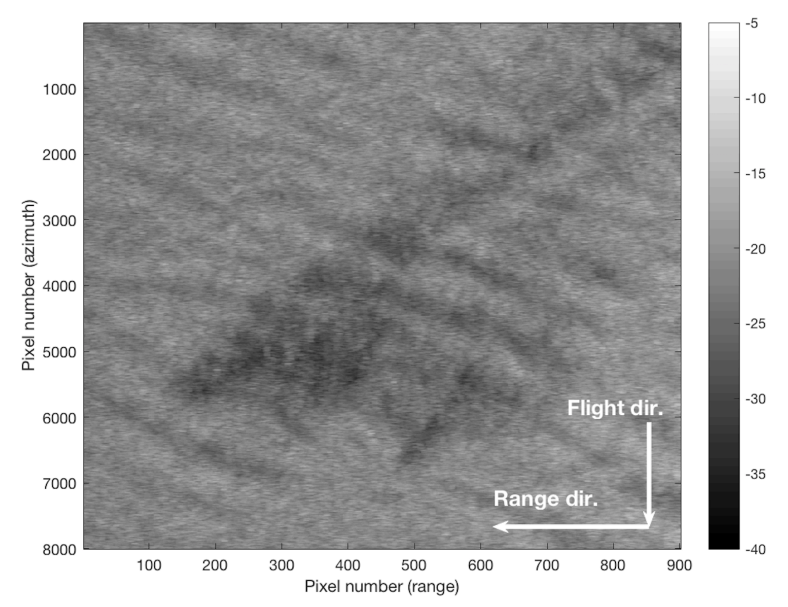

(b) L-band F-SAR scene 12 June 2019, 11:49 UTC.

Fig. 2 (a) Intensity images $\left(\sigma_{0 V V}[d B]\right)$ for the $\mathrm{A} 1 \mathrm{~L}$ acquisition, multi-looked by $9 \times 9$ window. The region-of-interest of the slick of emulsion is outlined with a red box while the region of interest of open ocean is outlined with a yellow box. (b) Same as in (a) but for the A2 L acquisition. DLR Data and Products (2019) - All Rights Reserved.

Table 1 summarizes the relevant information pertinent to the acquisitions as well as outlining the wind conditions that were present. In subsequent sections, the following notation will be adopted. The slick shown in Fig. 2 (a) will be referred to as A1 and the slick shown in Fig. 2 (b) will be referred to as A2. Specific frequency acquisitions will be delineated with the suffix -X, -S or -L. This indicates that A1X refers to the slick in the X-band acquisition of A1 and A1S refers to the slick in the S-band acquisition of A1 etc.

The mineral oil was discharged between 05:30 and 05:54 UTC. Between the times of 11:17 to $11: 30$ the mineral oil emulsion slick was estimated to have a surface area between 1.5 to $1.7 \mathrm{~km}^{2}$. 

MONTH 20XX

\section{Noise Analysis}

The utility of SAR for the purpose of marine surface slick characterization is greatly hindered by the presence of internal system noise of the sensor [17]. This background noise (or noise floor) of the sensor is referred to as the noise equivalent sigma zero (NESZ). In order to develop methodologies to correctly characterize the internal state of surface slicks, the NESZ must be lower than the measured NRCS of the scattering surface.

Current space-borne SARs have relatively high noise floors, rendering them impractical for applications where the crosspolarization information is required. As an example, Radarsat$2 \mathrm{~s}$ quad-polarization modes have NESZ values that lie between -27.5 and $-43 \mathrm{~dB}[18]$. Given the highly non-depolarizing nature of the ocean surface, sensors with low noise floors are required in order to utilize information that can be gained from the crosspolarization channels.

Fig. 3 (a) and (b) shows the NESZ curves for the X-, S- and L-band acquisitions in red, green and blue, respectively, for A1 and A2. This NESZ information was provided by DLR.

The solid lines represent a high-order polynomial fit to the average NESZ values, computed for all azimuth values, across range direction. The areas subtended by the dashed lines, show one standard deviation of these average NESZ values. The Xband NESZ curves show an anomalous 'hump' in the very near range. This is due to invalid samples in the extreme near range for that frequency band [personal communication DLR].

As can be seen, in both cases the L-band acquisition have significantly lower NESZ values ranging between -45 to $-60 \mathrm{~dB}$ approximately while both the X- and S-bands have NESZ values ranging between -30 to $-45 \mathrm{~dB}$ approximately. Note, the NESZ curves are shown for the entire length, in range direction, of the acquisition. The solid, vertical, black lines indicate the regions of interest that correspond to the oil slick regions shown in Fig. 2, i.e. the red box in Fig. 2 (a) and the entire image in Fig. 2 (b). As can be seen, these regions of interest correspond closely to the NESZ minimum for both the S- and L- band acquisitions but not for the $\mathrm{X}$-band acquisitions.

It should be noted that there is a high degree of correspondence for the NESZ curves shown for the X- and Sband acquisitions between Fig. 3 (a) and (b). However, when comparing the L-band NESZ curves, there appears to be a 3-4 $\mathrm{dB}$ difference between them. The reason for this difference is not fully known, but may either be due to a low level of radio frequency interference (RFI) or a small change in the internal instrument delay on the receive paths, causing a small additional differential delay between the HV and VH channels. Any mis-registration of the cross polar channels will degrade the NESZ [DLR, personal communication].

In order to determine the feasibility of using these 6 acquisitions (i.e. the X-, S- and L-band imagery in both $\mathrm{A} 1$ and A2), the following operations were carried out. The pixels that correspond to the surrounding ocean areas were masked out by hand, and then, following [19] and [4], a $6 \mathrm{~dB}$ threshold was imposed where by pixels (in the $\mathrm{VV}, \mathrm{HH}$ and $\mathrm{HV}$ polarization channels of each acquisition) that were greater than $6 \mathrm{~dB}$ above the noise floor were kept for further analysis, and pixels that were less than $6 \mathrm{~dB}$ above the noise floor were discarded. This
$6 \mathrm{~dB}$ threshold was imposed so as to ensure that only pixels that primarily contain signal are included in the analysis.

Fig. 3 (c) and (d) shows the pixels that correspond to $6 \mathrm{~dB}$ above and below the noise floor, highlighted in green and red, respectively for the A1L and A2L. As can be seen, a significant proportion of the pixels are above the threshold in the $\mathrm{A} 1 \mathrm{~L}$ acquisition, while all the pixels are above the threshold in the $\mathrm{A} 2 \mathrm{~L}$ acquisition. This is most likely due to the fact that $\mathrm{A} 1$ was acquired at a larger look angle than A2.

Fig. 3 (e) and (f) shows the pixels that are $6 \mathrm{~dB}$ above the noise floor for the two S-band acquisitions. As can be seen, a significant proportion of the pixels, internal to the oil slick, lie below the $6 \mathrm{~dB}$ threshold. For this reason, only the L-band acquisitions, $\mathrm{A} 1 \mathrm{~L}$ and $\mathrm{A} 2 \mathrm{~L}$ will be considered in the preceding sections.

The result of the noise analysis for the two X-band acquisitions are not shown, but yield similar results to the two S-band acquisitions.

Note, to make it easier for the reader, the specific acquisition that each individual image refers to in Fig. 3 is indicated in the bottom right. This convention is adopted throughout the paper.

\section{SENSITIVITY TO SURFACE ROUGHNESS}

Appendix B outlines an estimation procedure for the largescale surface roughness parameter $s$ by using method 1. In section II.B, it was also shown that by using method 2 a series of look-up charts can be constructed whereby the parameter $s$ and $|\varepsilon|$ can be determined simultaneously. The underlying assumption is that by utilizing the information contained within the co- polarization and cross- polarization channels, more accurate estimations for $|\varepsilon|$ can be performed since no assumptions about $s$ have to be made.

In this section, the differences in $s$ values that are obtained, when both methods are applied, are investigated. It is also investigated whether the box size for the estimation procedure in method 1 effects the estimation of $s$. It should be noted that [11] attempted to determine the effect of the dimensions of averaging window on the estimation of $s$, and found that average values were not affected but the variance of the scatter within each box was altered. For that reason, in this study an averaging box size of $9 \times 9$ pixels was chosen. The effects of these differences on the retrieval results of $|\varepsilon|$ are then demonstrated in a sensitivity analysis in the final subsection.

It should be noted that it is not expected to see large deviations in the large-scale surface roughness parameter $s$ within and without the sick filled areas as the presence of oil acts to primarily dampen the short-scale capillary waves [20].

\section{A. Difference in large scale roughness parameter $s$ obtained from both methods}

The values of $s$ that will be analyzed in this section, corresponding to the area of open ocean bounded by that yellow box in Fig. 2 (a), can be seen in Fig.4.

In order to determine the difference in retrieval results for the roughness parameter $s$ using the two methods, first A1S and A1L were divided into square regions of size $100 \times 100$ pixels 


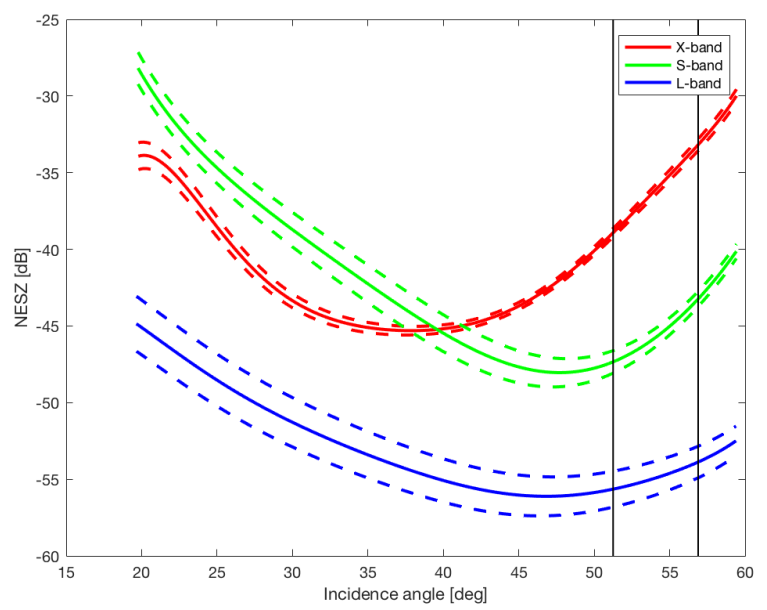

(a)

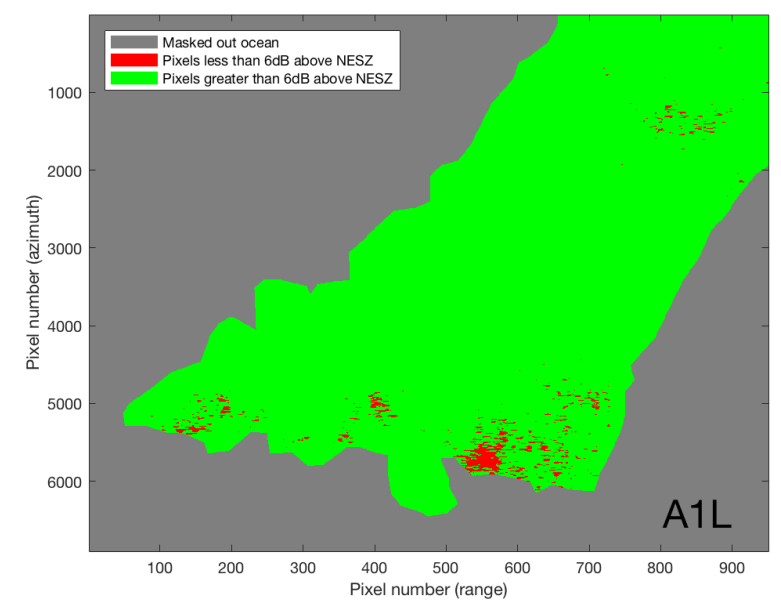

(c)

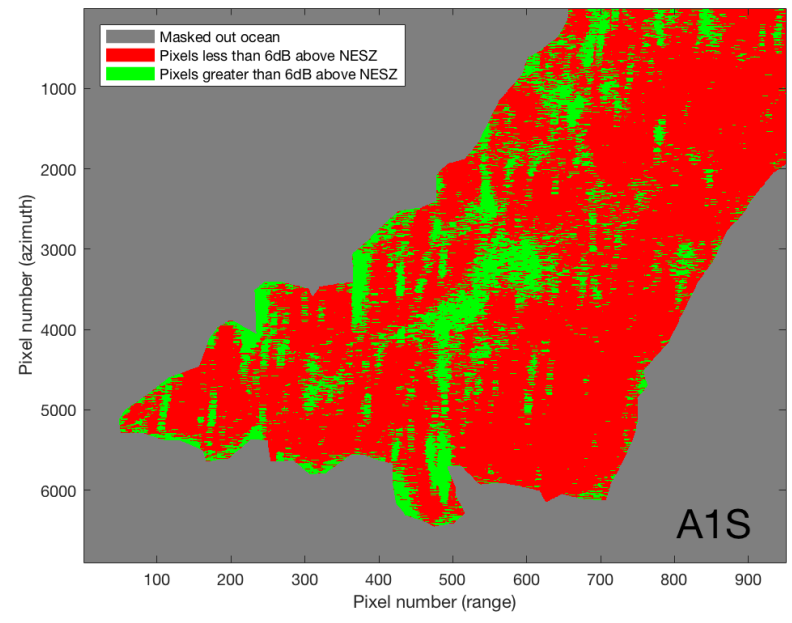

(e)

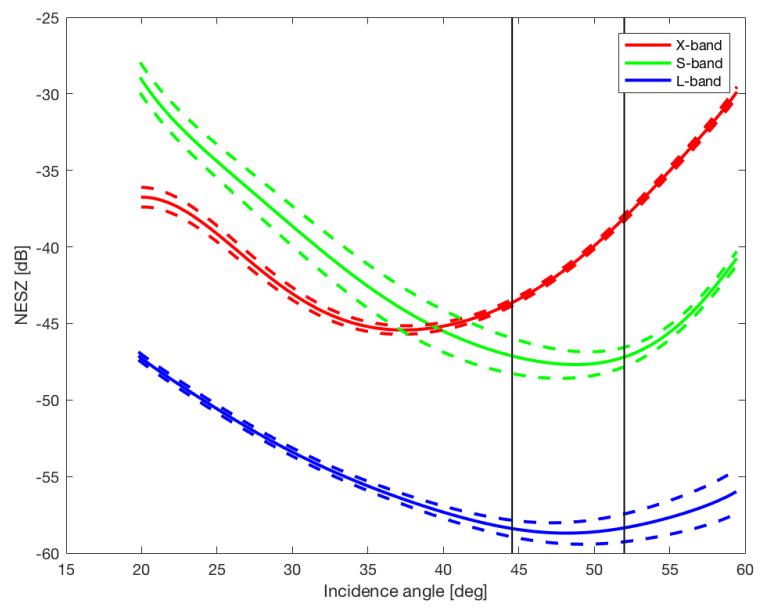

(b)

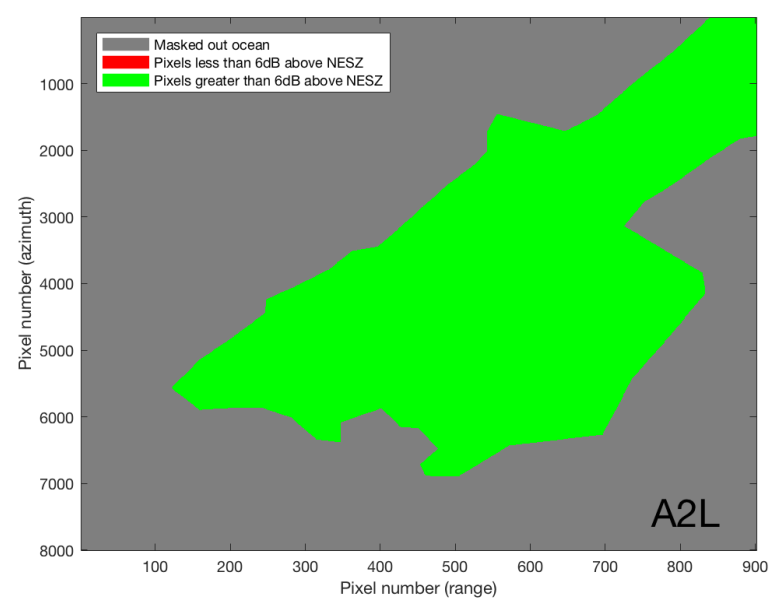

(d)

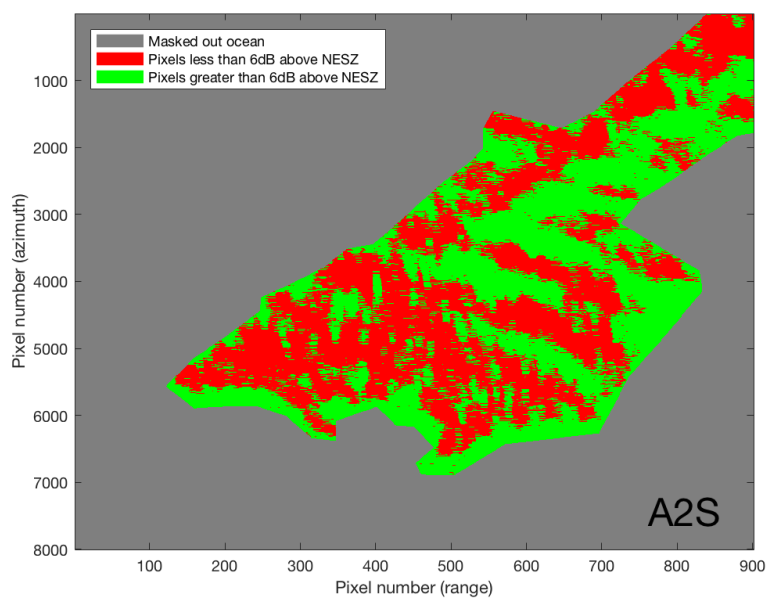

(f)

Fig. 3 (a)-(b) X-, S- and L-band NESZ curves for the entire A1 and A2 acquisitions. Vertical black bars denote the region of interest of the oil slick (see Fig. 2). The areas subtended by the dashed lines, show one standard deviation of the average NESZ values. (c)-(d) Pixels that have values greater than $6 \mathrm{~dB}$ above the NESZ for A1L and A2L are highlighted in green. Pixels that have values less than $6 \mathrm{~dB}$ above the NESZ are marked in red (e)-(f) Pixels that have values greater than $6 \mathrm{~dB}$ above the NESZ for A1S and A2S are highlighted in green and red for pixels less than $6 \mathrm{~dB}$ above the NESZ. 


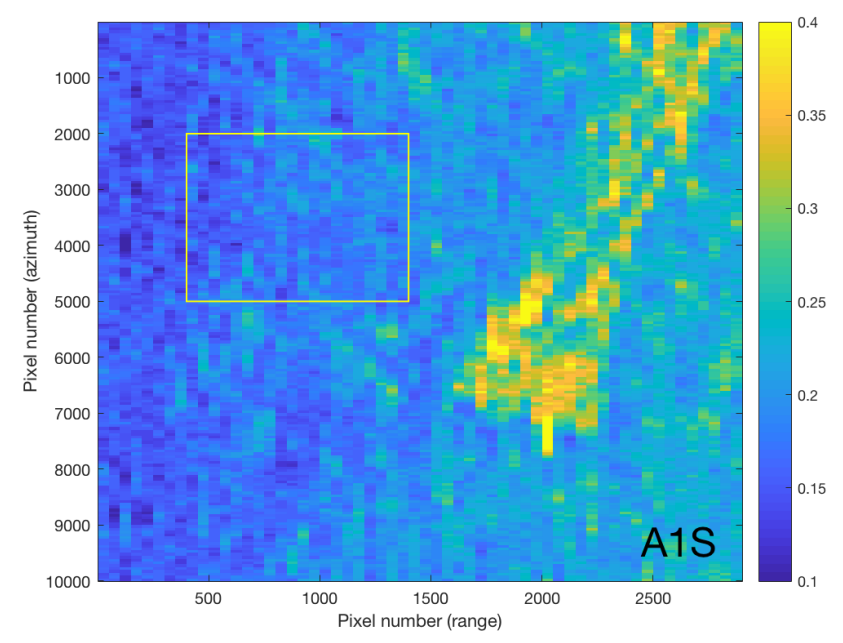

(a)

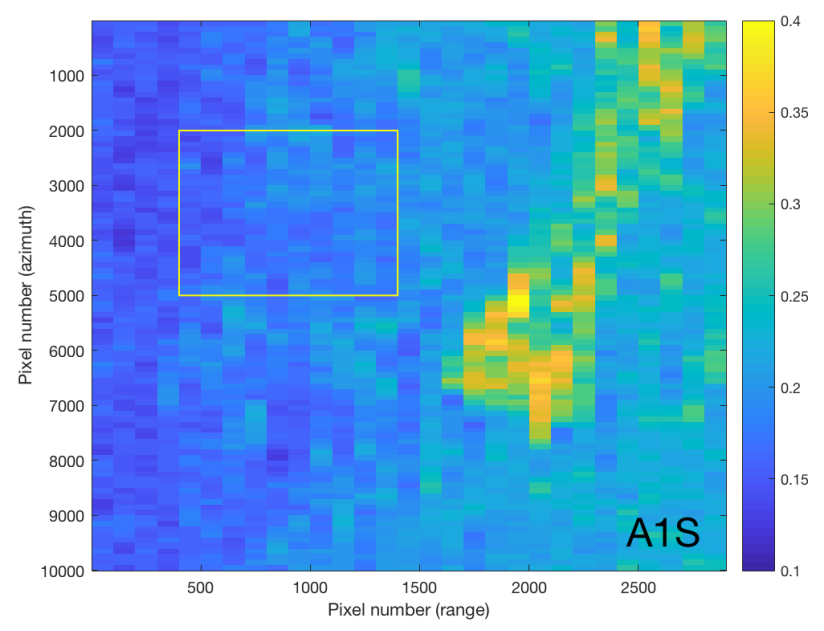

(c)

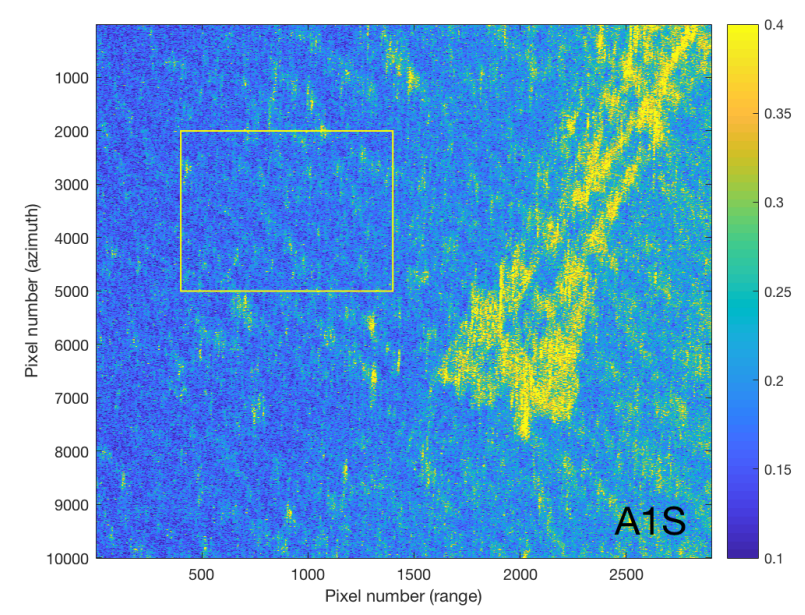

(e)

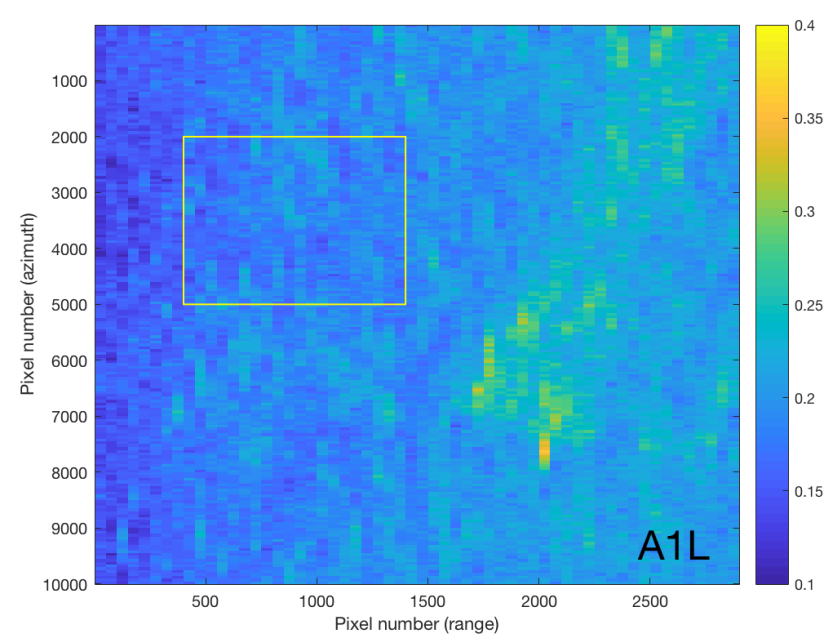

(b)

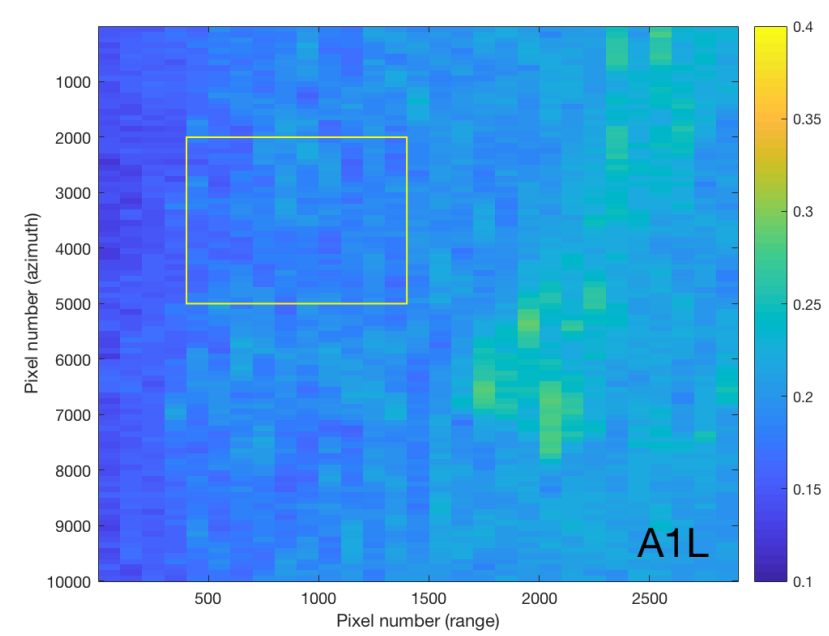

(d)

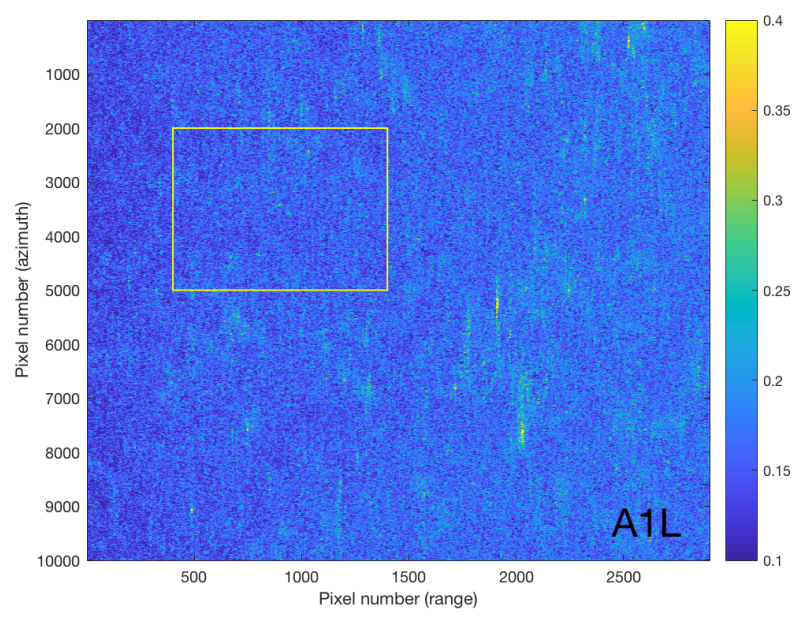

(f)

Fig. 4 (a)-(b) values for $s$ calculated within box regions of size $50 \times 50$ pixels for the acquisitions A1S and A1L, respectively using method 1 . (c)-(d) values for $s$ calculated within box regions of size $100 \times 100$ pixels for the acquisitions A1S and A1L, respectively, using method 1. (e)-(f) values for $s$ calculated by using method 2 for A1S and A1L, respectively. As can be seen, pixel-by-pixel values are obtained. The yellow box in all images outlines a region of interest of open ocean that will be used in future analysis. 
and $50 \times 50$ pixels each. Fig. 4 (a) and (b) show the $s$ values for A1S and A1L, respectively, computed using boxes with dimensions of $50 \times 50$ pixels. Fig. 4 (c) and (d) show the same, but computed using boxes with dimensions of $100 \times 100$ pixels.

As can be seen, the areas that correspond to the oil slick in A1S show higher values for $s$ than the A1L with both cases showing higher values for $s$ within slick than the surrounding open ocean. The reason why the values for $s$ are higher within slick when compared to outside, is that when estimating values for $s$ within slick, the expectation for the co-polarization scatter from individual square regions are still calculated at a nominal value for the $|\varepsilon|$ of open water. Given that the co-polarization ratio will have lower values within slick, this naturally leads to higher values for $s$. This can be seen from Fig. 13 (a) in Appendix B. For this reason, it is necessary to estimate $s$ within slick from the surrounding ocean areas, when only the copolarization data is available, or when employing X- or Sbands. It should be noted that the lower $s$ values within slick in $\mathrm{A} 1 \mathrm{~L}$, for both the $50 \times 50$ pixel and $100 \times 100$ pixel case, are most likely due to larger contributions from the surface roughness, than alterations to the dielectric constant of the scattering surface, due to the longer wavelength of the incoming radiation in L-band when compared to S-band, i.e. in this case the slick is behaving more like a thin film and is not decreasing the effective dielectric constant of the ocean surface because of the longer wavelength at L-band.

Fig. 4 (e) and (f) show the $s$ values as they are retrieved by using method 2 for A1S and A1L, respectively. As can be seen, pixel-by-pixel values are obtained. In the A1S case, in Fig. 4 (e), values within slick are higher than outside slick. In this case, this is due to the high noise corruption in the HV channel. This was shown in the noise analysis in Section V. For the A1L case, a homogenous field of $s$ values is obtained. This is because by running the full model we are able to retrieve independent estimations of the surface roughness and the dielectric constant of the scattering surface due to the high signal-to-noise ratio of all polarimetric channels for the $\mathrm{A} 1 \mathrm{~L}$ case.

Each $50 \times 50$ pixel or $100 \times 100$ pixel box within the yellow region of interest in Fig. 4 (a)-(d) represents a single local estimate for $s$. The histogram for the collection $s$ values that corresponds to each one of these box areas in Fig. 4 (e) and (f) was computed and the expectation value was determined from each histogram. The distribution of $s$ values resembled a gamma shaped distribution with a very light tail. For this reason, the numerical average was used as an estimation for the expectation. The difference between the expectation for the $s$ values determined from the histogram of values in Fig. 4 (e) and (f) and the box regions in Fig. 4 (a)-(d) was determined. The histograms of these difference values are shown in Fig. 5.

Fig. 5 (a) shows the case for A1S and Fig. 5 (b) shows the case for A1L. As can be seen typical values are around 0.030.04 . Note for the $50 \times 50$ box case, there is a higher degree of variance. This is to be expected as the $50 \times 50$ box case resulted in a smaller number of samples, which results in greater variability. However, in both cases for A1S and A1L the expectations remain the same.

These results show that by employing method 1 the $s$ values are overestimated, with typical values ranging from 0.02 to

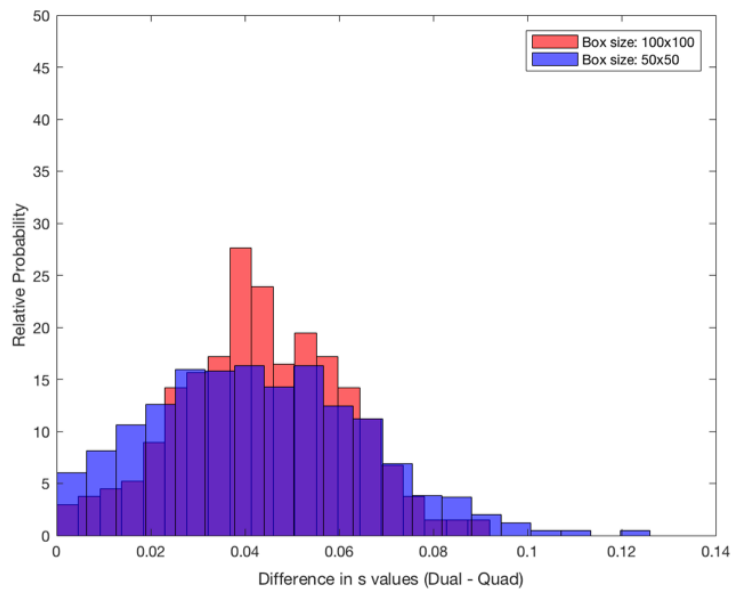

(a) A1S difference values

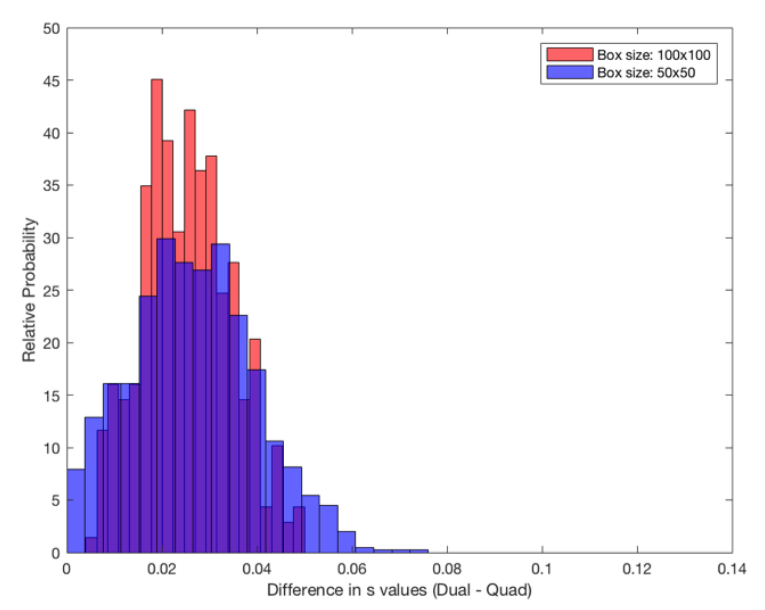

(b) A1L difference values

Fig. 5 (a)-(b) Histograms of difference values of $s$ for the pixels subtended by the yellow region of interest in Fig. 4. Values were calculated by determining an $s$ value for each box region, determined by using just the co-polarization ratios, and subtracting from it an expectation value for the distribution of $s$ values corresponding to each square region that are determined by using both polarizations ratios. Note, the overlap between the two has a slightly purple appearance.

0.06 . In the next subsection, a sensitivity analysis that is designed to determine what the effects on the retrieval results of $|\varepsilon|$, when $s$ is perturbed by a small amount, which is meant to simulate the uncertainty in $s$, is presented.

\section{B. Sensitivity Analysis}

Fig. 6 shows the PTSM co-polarization ratio plotted against $|\varepsilon|$ for a value of $s$ equal to 0.2 (solid line). A $\Delta s$ value, hereinafter referred to as a perturbation, equal to 0.02 , was added to the original value for $s$. The PTSM co-polarization ratio was then again plotted again against $|\varepsilon|$, with a value for $s$ equal to 0.22 (the original $s$ value plus the perturbation value) and can be seen in Fig. 6 (dashed line). 


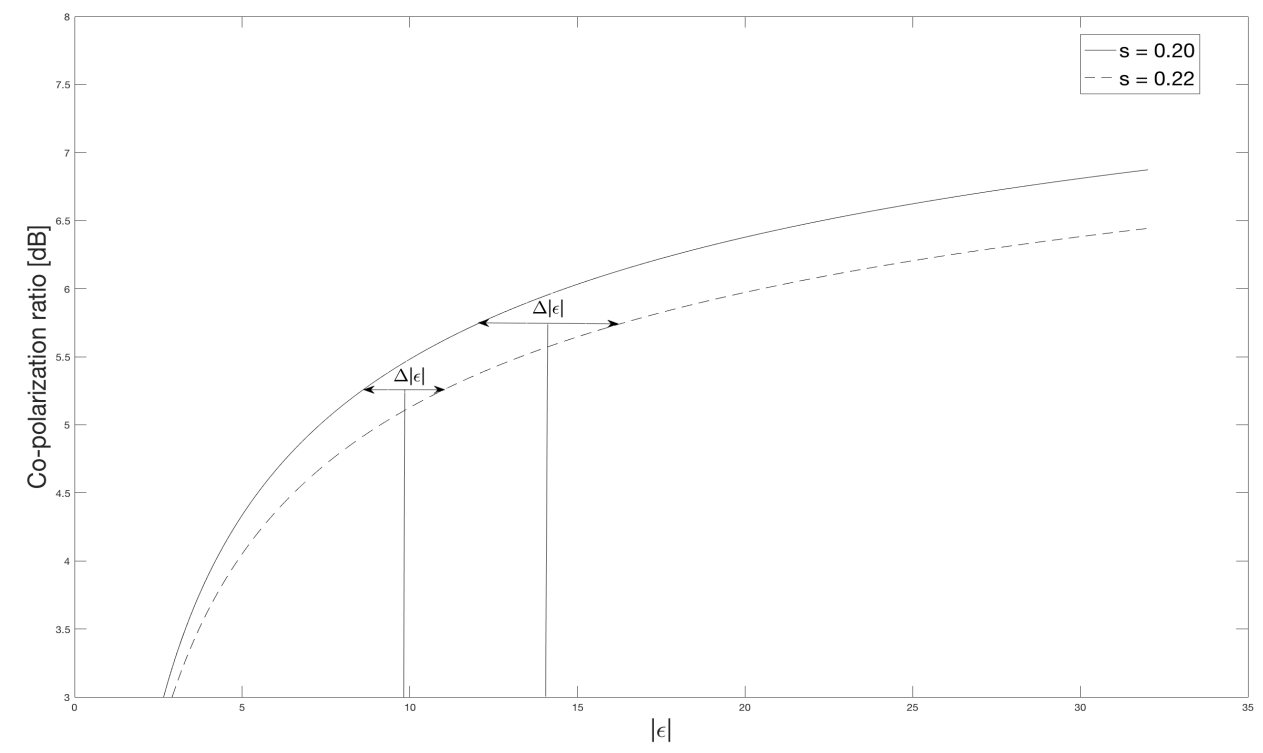

Fig. 6 Method for assessing the sensitivity of the co- polarization PTSM values to small perturbations in values of $s$. A PTSM curve is plotted with a specific value for $s$ equal to 0.2 . A small perturbation of 0.02 is added to the original $s$ value. The difference in $|\varepsilon|$ i.e. $\Delta|\varepsilon|$ is recorded for successively higher values of $|\varepsilon|$. The curves shown above were computed for $H_{t}=0.9, v=40^{\circ}$ and radiation frequency of $3.25 \mathrm{GHz}$.

The difference in $|\varepsilon|$ values retrieved from the two curves is illustrated, and as can be seen, grows with increasing $|\varepsilon|$. This difference is delineated by the value $\Delta|\varepsilon|$. A central $|\varepsilon|$ value, for the quantity $\Delta|\varepsilon|$ is marked by the vertical black line.

In order to illustrate the effect of these perturbations on the retrieval results for $|\varepsilon|$, the PTSM co-polarization ratio was computed for $s$ values equal to 0.1 and 0.22 . These values were chosen as they represent typical lower and upper limits for the surface facet slopes on the ocean surface, respectively. A set of four perturbation values were chosen equal to $0.01,0.02,0.03$ and 0.04 . These perturbation values were then added to the original $s$ values of 0.1 and 0.22 , and the PTSM co-polarization curves were then calculated again

The $\Delta|\varepsilon|$ values along with the central $|\varepsilon|$ values (as illustrated in Fig. 6) were determined. Fig. 7 show scatter plots of the retrieved $\Delta|\varepsilon|$ values plotted against the central $|\varepsilon|$ values, computed with inputs for wavelength in the model, equal to the sensing wavelength of the F-SAR instrument in X-, S-, and Lbands. In order to generalize the results, the incidence angle parameter was chosen randomly from a uniform distribution ranging between $35^{\circ}$ and $55^{\circ}$. The scatter for each perturbation is plotted and color coded as either red, green, blue or black. As can be seen, the value for the inputted perturbation value $(\Delta s)$ results in the most variation in $\Delta|\varepsilon|$ as opposed to other input parameters such as sensor wavelength or incidence angle.

It can also be seen however that for low values of $|\varepsilon|, \Delta s$ values result in small values for $\Delta|\varepsilon|$. This implies that the PTSM co-polarization ratio, is robust to uncertainty in $s$ for low dielectric values but this uncertainty grows with increasing $|\varepsilon|$.

The implication of this, is that we should expect to see similar results for $|\varepsilon|$ when the PTSM is applied using both methods at lower dielectric values within oil slick, with a divergence occurring at higher dielectric values.

\section{RESUlTS}

Fig. 8 (a) and (b) show the inversion results, i.e. $|\varepsilon|$ values, acquired from using method 1 and method 2, for the A1L acquisition, respectively. Fig. 8 (c) and (d) show the same, but for the A2L acquisition.

When comparing the inversion results obtained from the two methods, it is easy to see that similarities exist. This is in relation to the presence of internal zones of low $|\varepsilon|$ values (approximately ranging between 2 - 8) that exist within both sets of imagery.

Where the two sets of imagery diverge, is in relation to intermediate values for $|\varepsilon|$ (approximately $8-15$ ). In the results obtained from just using method 1 a higher proportion of pixels with $|\varepsilon|$ values in this range are observed within slick, whereas in the results obtained using method 2, corresponding pixels have lower values.

This can be explained in the context of the sensitivity analysis from section IV.B. As illustrated, lower values for $|\varepsilon|$ will be robust to uncertainties in values for $s$, with larger errors in the estimation of $|\varepsilon|$ occurring at higher dielectric values.

Given that values of $s$ tend to be overestimated when using method 1 (this is shown in Fig. 5) and that higher values of $s$ will result in higher estimates for $|\varepsilon|$ (this is illustrated in Fig. 13 (a)), the higher proportion of high $|\varepsilon|$ values present in the inversion results in Fig. 8 (a) and (c) are explained. 
Fig. 9 (a) and (b) show binned scatter plots with $|\varepsilon|$ values retrieved from using method 1 on the $\mathrm{x}$-axis and $|\varepsilon|$ values retrieved from using method 2 on the $\mathrm{y}$-axis for $\mathrm{A} 1 \mathrm{~L}$ and $\mathrm{A} 2 \mathrm{~L}$, respectively. As can be seen for values of $|\varepsilon|$ ranging between 2 to 8 approximately, a high degree of correspondence can be seen between the two sets of results, with the method 1 results being over estimated for higher $|\varepsilon|$ values in comparison to the results obtained when using both the co- and cross- polarization information.

In order to test the method of determining $|\varepsilon|$ by using just the co-polarization ratio i.e. method 1, the $s$ values that were obtained from applying method 2 (shown in Fig. 4 (f) for the case of A1L) were inserted into the PTSM co- polarization ratio and estimations for $|\varepsilon|$ were found. The expectation is that the $|\varepsilon|$ values obtained would be equivalent for both methods. The scatter plots shown in Fig. 9 (c) and (d), which again show binned scatter plots with $|\varepsilon|$ values retrieved form utilizing the method 1 on the $\mathrm{x}$-axis and $|\varepsilon|$ values retrieved from utilizing method 2 on the $y$-axis for $\mathrm{A} 1 \mathrm{~L}$ and $\mathrm{A} 2 \mathrm{~L}$, respectively are shown. As can be seen, the values obtained are similar with the majority laying on the red, diagonal line. This indicates that both methods are equivalent with alterations in $s$ being the main driver for errors occurred.

Given that F-SAR has a low noise floor, but that a high degree of noise corruption may occur in the cross-polarization channels for certain frequency bands over oil slick, e.g. X- and S-bands in this study, as illustrated in section III.C, the possibility of obtaining very accurate estimations for $s$ from the surrounding ocean is investigated.

The $s$ values that were derived from utilizing the entire model, an example is shown in Fig. 4 (f) for the case of A1L, were derived. The area that corresponds to the oil slick was masked out, and then inwards interpolation (the same procedure applied to the estimations of the $s$ values that are derived from just using the co-polarization ratio as outlined in Appendix B) was performed. Note, the interpolation procedure employed here smoothly interpolates inwards from pixel values at the outer boundary areas of a masked out region of interest by computing the discrete Laplacian over the region, and then solving the Dirichlet boundary value problem.

Estimates for $|\varepsilon|$ were then determined and compared to the $|\varepsilon|$ inversions results obtained from using both the co- and cross-polarization information. Scatter plots for the A1L and A2L acquisitions can be seen in Fig. 9 (e) and (f). As can be seen, for low values of $|\varepsilon|$, there is still a high degree of correspondence between the inversion results. Interestingly, for higher values of $|\varepsilon|$, greater than 8 , the correspondence is retained somewhat with less divergence than in the case of Fig. 9 (a) and (b).

In order to determine the degree of correspondence between each of the cases displayed in Fig. 9, first the correlation coefficient was calculated over the entire range of values, i.e. ranging between values of $|\varepsilon|$ of $2-20$ and then between the ranges of $2-8,8-15$ and finally $15-20$. The results are displayed below in Tables 2 and 3. For ease of reference, the values are cited in terms of the panel in Fig. 9 for which they correspond.

With the exception of the results displayed in Fig.9 (c) and (d), strong moderate correlation values are observed for values of $|\varepsilon|$ between $2-8$ with the degree of correlation dropping for the intervals of $|\varepsilon|$ between $8-15$ and $15-20$.

Table 2: Values for the correlation coefficient calculated for the results displayed in Fig. 9 for the A1L acquisition

\begin{tabular}{|l|c|c|c|c|}
\hline & $\mathbf{2 - 2 0}$ & $\mathbf{2 - 8}$ & $\mathbf{8 - 1 5}$ & $\mathbf{1 5 - 2 0}$ \\
\hline Fig. 9 (a) & 0.51 & 0.58 & 0.20 & 0.02 \\
\hline Fig. 9 (c) & 0.99 & 0.98 & 0.98 & 0.92 \\
\hline Fig. 9 (e) & 0.58 & 0.59 & 0.35 & 0.07 \\
\hline
\end{tabular}

Table 3: Values for the correlation coefficient calculated for the results displayed in Fig. 9 for the A3L acquisition

\begin{tabular}{|c|c|c|c|c|}
\hline & $\mathbf{2 ~ - ~ 2 0}$ & $\mathbf{2 ~ - ~ 8 ~}$ & $\mathbf{8 ~ - ~ 1 5}$ & $\mathbf{1 5}-\mathbf{2 0}$ \\
\hline Fig. 9 (b) & 0.45 & 0.53 & 0.18 & 0.04 \\
\hline Fig. 9 (d) & 0.99 & 0.78 & 0.95 & 0.92 \\
\hline Fig. 9 (f) & 0.54 & 0.50 & 0.34 & 0.06 \\
\hline
\end{tabular}

\section{COMPARISON TO IN-SITU DATA}

In this section we will attempt to link the values for $|\varepsilon|$, obtained via the PTSM, with the method outlined in section II.B for the $\mathrm{A} 1 \mathrm{~L}$ and $\mathrm{A} 2 \mathrm{~L}$ acquisitions, to aerial photography of the mineral oil emulsion slick. Fig.10 shows a photograph taken from the Dornier DO228-212 aircraft. The mineral oil emulsion slick can be seen as well as the soybean oil slick. The corresponding S-band SAR image is shown as well for reference. As can be seen, the "tail" of the mineral oil emulsion slick is visible in both sets of images emanating from the "head" of the slick in the middle background of both images to the right background. The photograph was taken at 10:46 UTC while the SAR acquisition was taken at 11:09 UTC. Note, aspects of this image may appear slightly blurry due to the fact that it was taken from the inside of the aircraft through glass.

Despite the fact that the "tail" of the slick is visible in the aerial photograph of Fig, 10. It is not observed from a less oblique viewing angle. Fig. 12 (a) shows the mineral oil emulsion slick as photographed from the Dornier DO228-212 aircraft. As can be seen, the "tail" of the slick is less apparent. Again, this image may appear slightly blurry due to the fact that it was taken from the inside of the aircraft through glass. Fig. 12 (b) shows Fig. 12 (a) after processing for the purposes of aiding interpretation. Specifically, the bottom $1 \%$ and top $1 \%$ of each color band was saturated, thus increasing the contrast of the image. In this image, the red zones indicate thicker regions within the slick in contrast to the yellow zones. In both Fig. 12 (a) and Fig. 12 (b) the three individual patches of oil are labeled as 1, 2 and 3. Fig. 12 (c) and (d) show the SAR acquisitions A1L and A2L. As can be seen, the three patches that are apparent in Fig. 12 (a) and (b) can be made out and are also numbered. A1L was acquired 27 minutes before the photograph of Fig. 12 (a) and F2L was acquired 19 minutes after the photograph of Fig. 12 (a).

Fig. 12 (e) and (f) show the inversion results for $|\varepsilon|$ reproduced from Fig. 8 (b) and (d). The results shown in Fig. 12 (e) show a greater concentration of lower $|\varepsilon|$ values concentrated in the "head" of the slick. The greatest concentration of low $|\varepsilon|$ values can be seen around the patches labeled 1 and 3 in Fig. 12 (c). When comparing the $|\varepsilon|$ values 
for A2L, as seen in Fig. 12 (f), with the aerial photograph shown in Fig. 12 (a) and (b), again concentrated areas of $|\varepsilon|$ values that correspond to the slick patches labeled 1 and 3 , can be distinguished.

As can be seen from Fig. 12 (a) the three patches of slick appear to belong to code 5 of the BAOAC. This is due to the fact that the slick appears to have a true color, reddish appearance surrounded by metallic, gray areas. According to BAOAC the reddish areas will have thickness greater than 200 $\mu \mathrm{m}$ while the grayish areas will have thicknesses between $5 \mu \mathrm{m}$ $-50 \mu \mathrm{m}$.

Fig 11 (a) and (c) show two photographs that were taken from the research vessel R/V Helmer Hanssen of the mineral oil emulsion slick. The green square in both images indicates a portion of the photograph that is displayed in Fig. 11 (b) and (d). In both Fig. 11 (a) and (c) the oil surface primarily shows a discontinuous true color appearance which corresponds to a Bonn code of 4. According to [21], this implies that the slick contains thickness regimes between 50 to $200 \mu \mathrm{m}$. Fig. 11 (b) shows a dark patch of oil slick from Fig. 11 (a). As can be seen this oil patch has a primarily continuous, dark color. This corresponds to a Bonn code of 5 which indicates that the slick contains thickness regimes greater than $200 \mu \mathrm{m}$. Fig 11 (d) shows a portion of Fig 11 (c) bounded by the green box. As can be seen the slick surface here appears to be highly textured with evidence of tar ball formation. Given that the textured surface can be seen on a photograph, areas of the slick are expected to have thickness reaching roughly $1 \mathrm{~mm}$. It should be noted that the BAOAC code 5 does not describe an upper limit to the oil slick thickness within this category. Only the lower limit of 200 $\mu \mathrm{m}$.

At two points of the day of 12 June 2019, six thickness measurements were made of the mineral oil emulsion slick, 4 at 13:30 UTC approximately and 2 at 17:15 UTC approximately. This was done by collecting oil samples by lowering a glass tube into the slick. The oil thickness of the samples was determined later in a laboratory by comparing them to reference samples. The three thickest samples, had thickness measurements of $200-320 \mu \mathrm{m}$ and were all measured around 13:30 UTC. The fourth sample measured around 13:30 UTC had a thickness of $160 \mu \mathrm{m}$. The thinnest thickness measurements were 180 and $20 \mu \mathrm{m}$ and were measured around 17:15 UTC. This confirms the observations made from the photos in Fig. 12 (a) and Fig. 11 that the slick was of a BAOAC code 5 and suggests that there may have been regions within the slick that had thickness of up to $1 \mathrm{~mm}$.

\section{CONCLUSION}

In the first part of this paper we presented the PTSM as well as two methodologies for determining $|\varepsilon|$, based on the amount of polarimetric information available. We have also introduced an experimental data set that was acquired during the NORSE 2019 oil-on-water campaign conducted in the North Sea, which contains multi-temporal F-SAR backscatter measurements of controlled discharges of mineral oil emulsion obtained in X-, Sand L-band. A signal-to-noise analysis of the data concluded

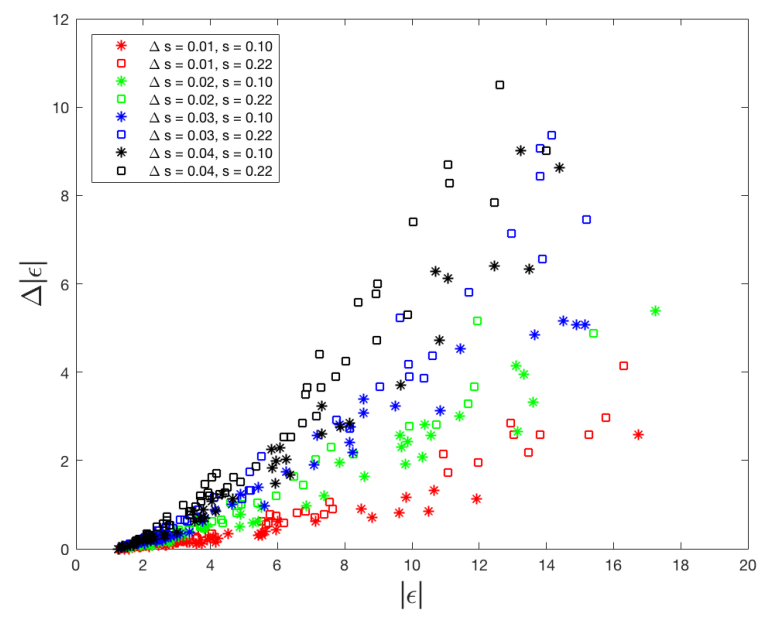

(a)

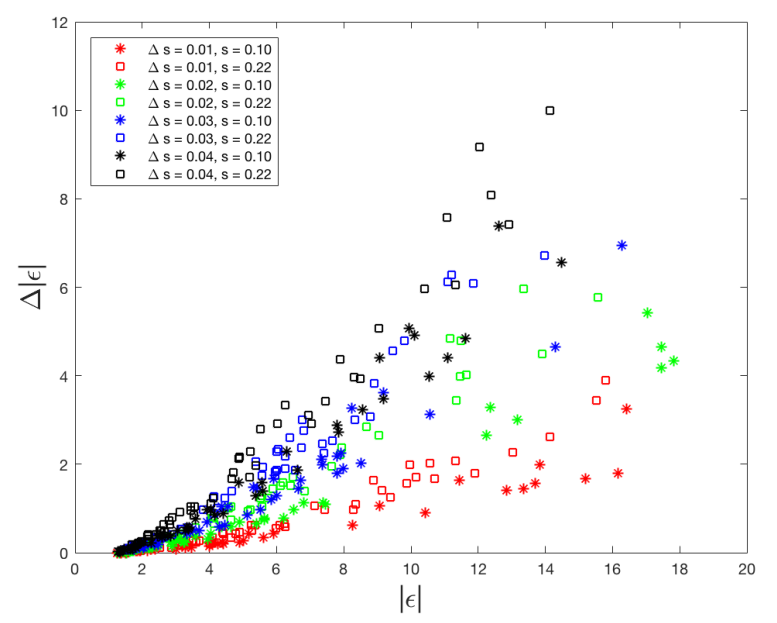

(b)

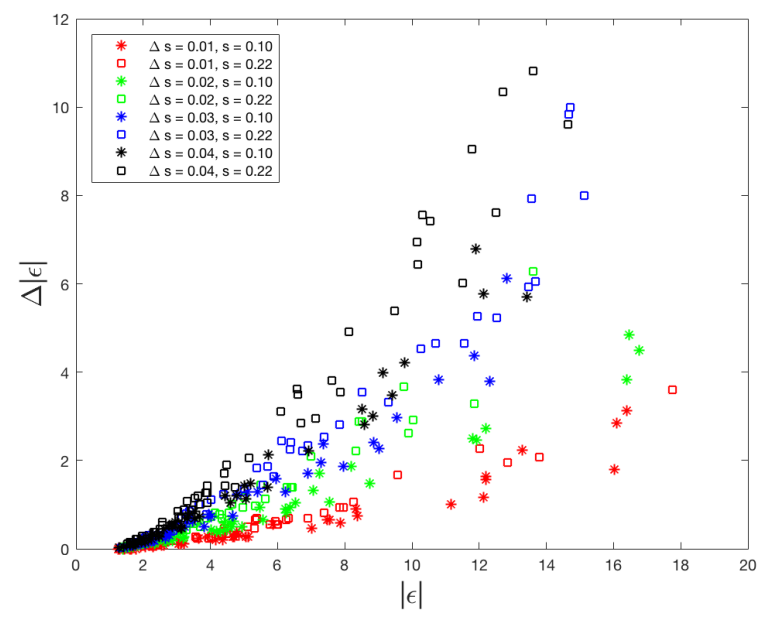

(c)

Fig. 7 (a)-(c) Results of sensitivity analysis of X-, S- and L-band, respectively. 


\section{MONTH 20XX}

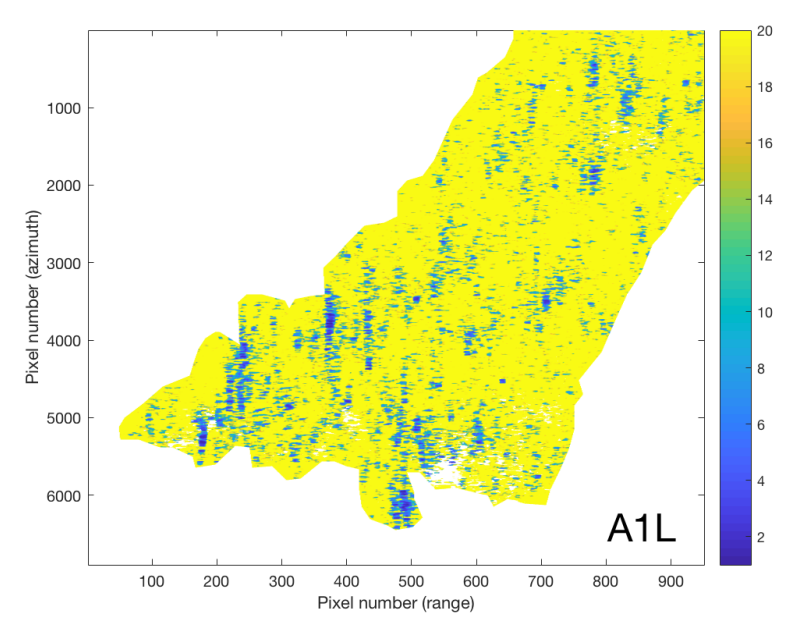

(a)

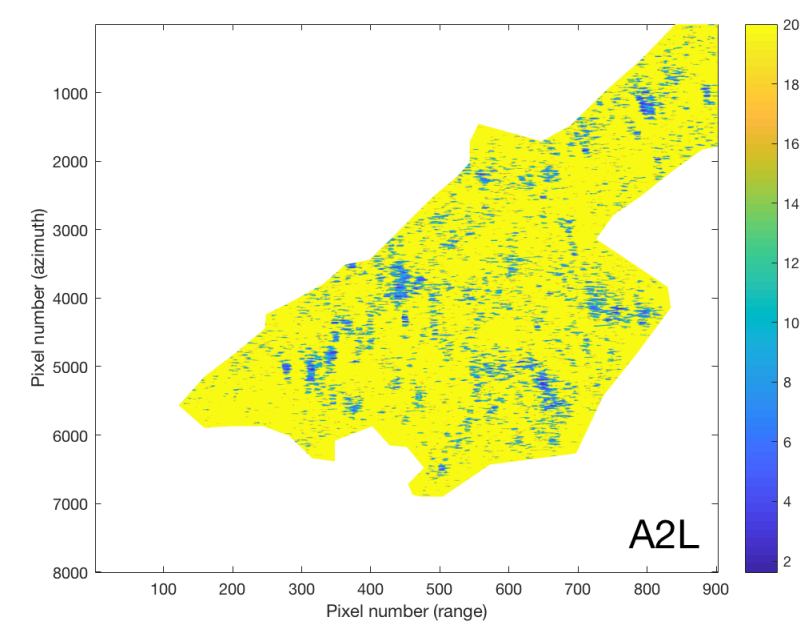

(c)

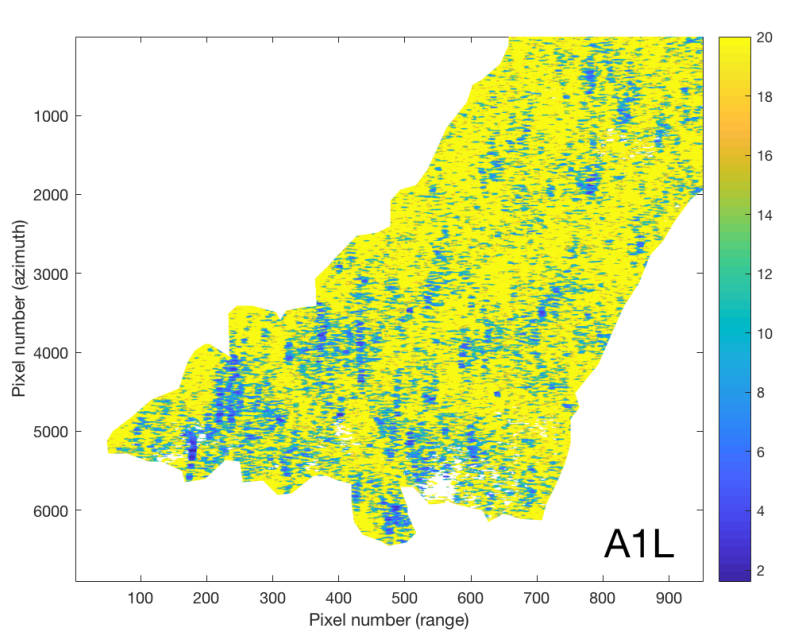

(b)

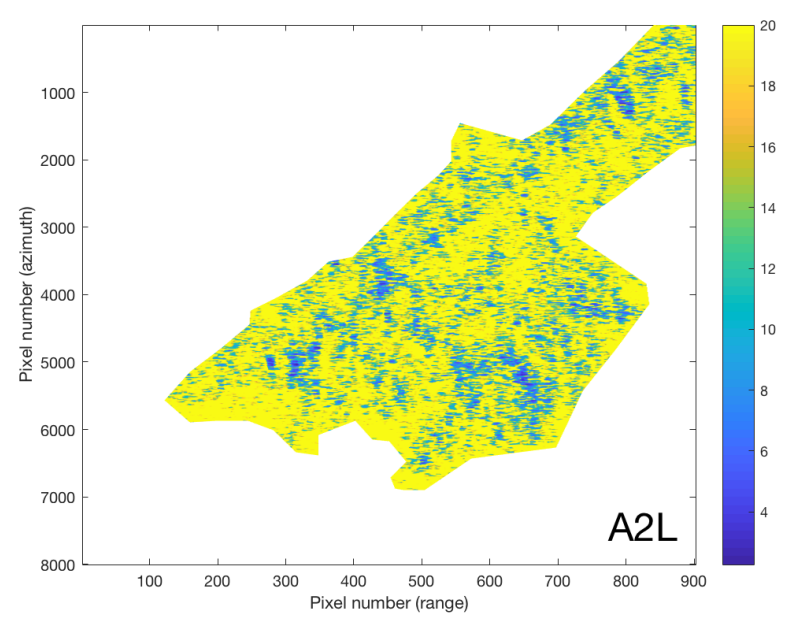

(d)

Fig. 8 (a) Values for $|\varepsilon|$ found after inversion for the slick of emulsion contained in A1L obtained by using method 1. (b) Inversion results for the slick of emulsion contained in A1L obtained by using method 2. (c) Inversion results for the slick of emulsion contained in A2L obtained by using method 1 (d) Inversion results for the slick of emulsion contained in A2L obtained by using method 2 .

that only the L-band data over the oil slick was suitable for use in this study.

In the second part of this paper, we explored the effect small errors in the estimation of the roughness parameter $s$ has on the inversion results for $|\varepsilon|$. First, we demonstrated that values for $s$ obtained from using method 1 are consistently overestimated when compared to the values of $s$ obtained from using method 2. A sensitivity analysis revealed that for low values of $|\varepsilon|$, uncertainties in $s$ do not lead to significant errors in the estimation for $|\varepsilon|$, but that errors increase for higher dielectric values.

This was demonstrated by examining the inversion results from $\mathrm{A} 1 \mathrm{~L}$ and $\mathrm{A} 2 \mathrm{~L}$. Both these acquisitions were inverted for $|\varepsilon|$ using both methods outlined in section II.B and Appendix B.
The results, as seen in Fig. 8, show that the lowest values for $|\varepsilon|$ were preserved across both methods employed. The underlying assumption made in this study is that values obtained from employing method 2 will yield more accurate results, since it is not required to make assumptions in regards to the roughness parameter $s$.

A brief comparison to in-situ data, collected in the form of IR aerial imagery, suggests that retrieval of $|\varepsilon|$ values, is able to accurately indicate areas of slick, where more oil is present. However, comparison between only one scene of an IR acquisition and one inversion result, both acquired one hour apart from each other, was made, indicating that more work is needed to verify if the PTSM is able to accurately characterize the dielectric properties of oil slick. In addition, the period in 


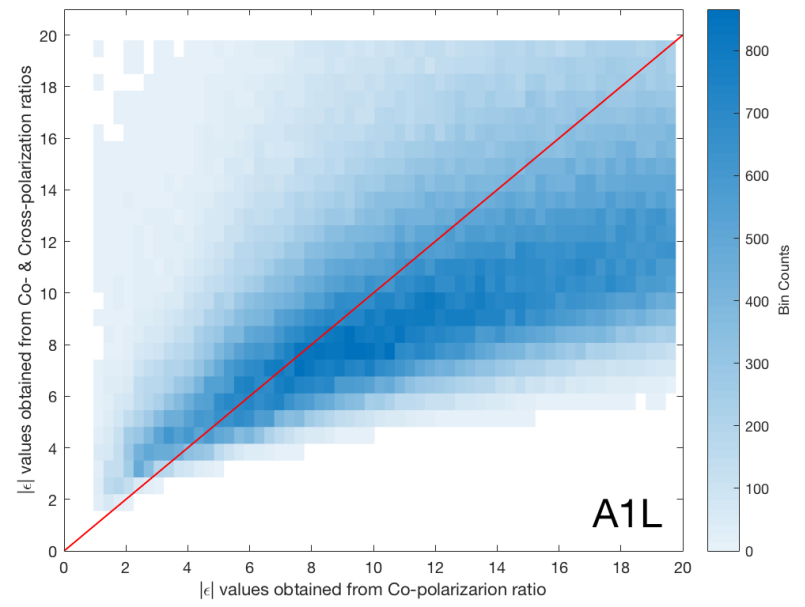

(a)

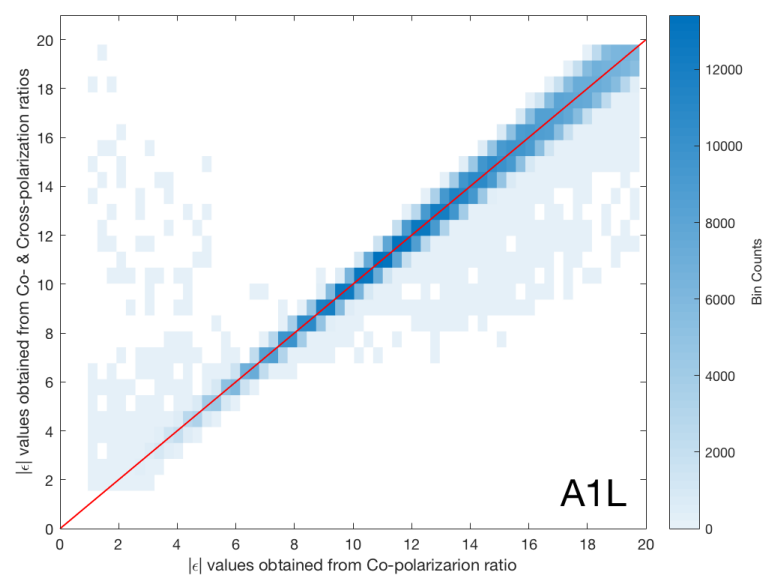

(c)

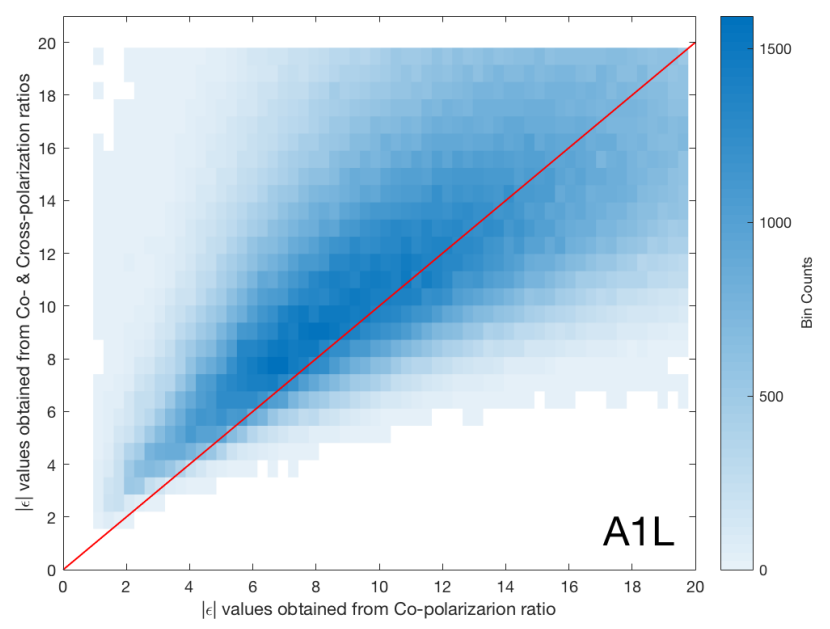

(e)

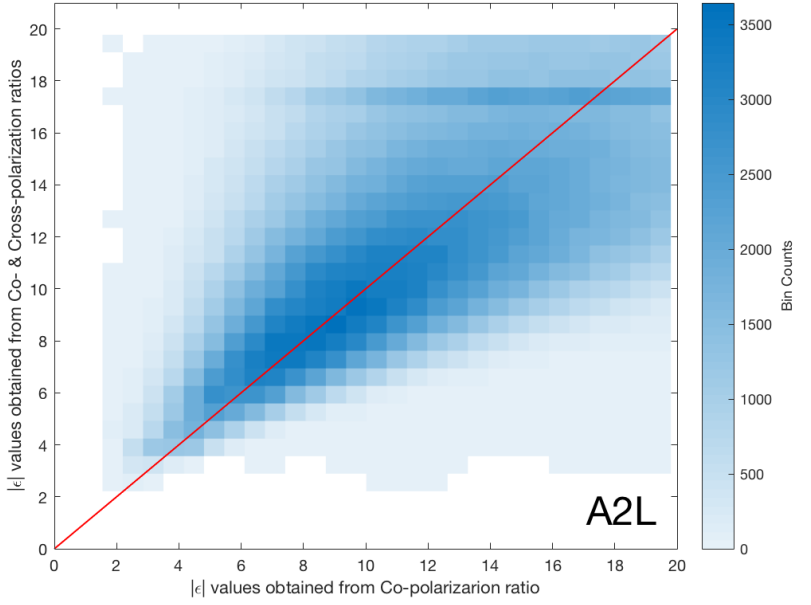

(b)

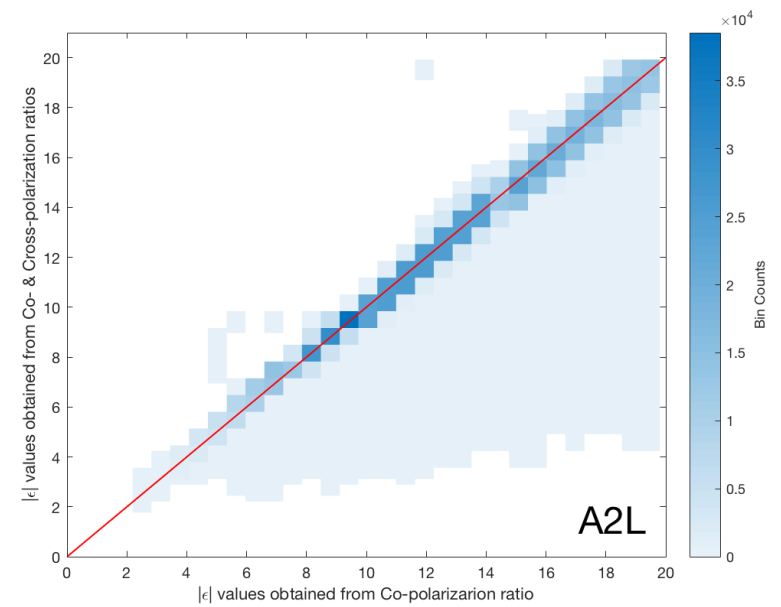

(d)

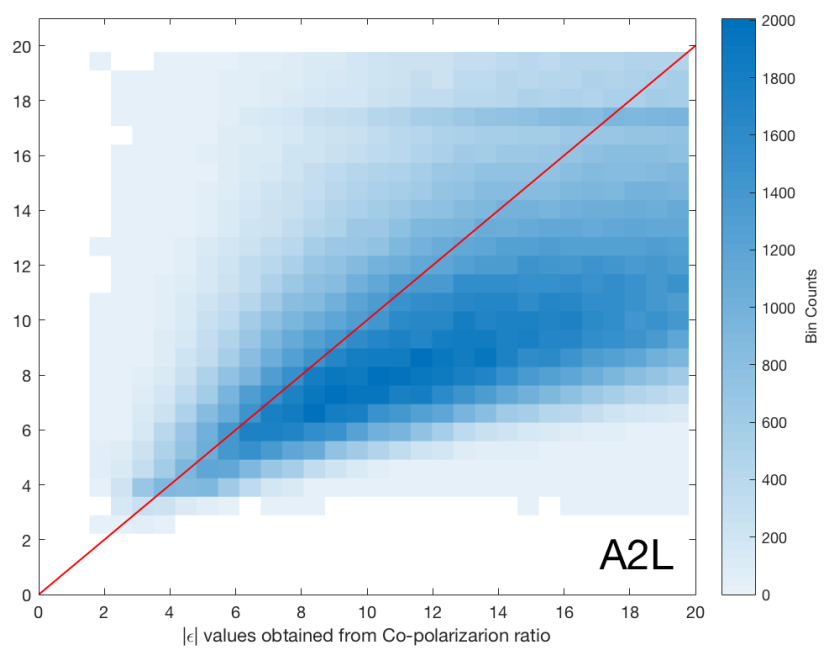

(f)

Fig. 9 (a)-(b) Scatter plots of $|\varepsilon|$ inversion values obtained from using method 1 on the x-axis vs. using method 2 on the y-axis for the slicks found in A1L and A2L, respectively. (c)-(d) show the same as the respective plots in (a)-(b) but with $s$ values obtained from using the co- and cross-polarization ratios (method 2) used in the method that just relies on the co- polarization ratio (method 1). (e)-(f) show again the same as the respective plots in (c)-(d) but the $s$ values that were obtained from method 2 were masked out over the slick areas, and then inwards interpolation was performed to estimate the $s$ values from the surrounding areas outside the slick. The resulting $s$ values were then fed into the PTSM using only the co-polarization ratio (method 1). The red line in all the 6 plots indicates the pixels which have a same $|\varepsilon|$ values obtained using both methods. 

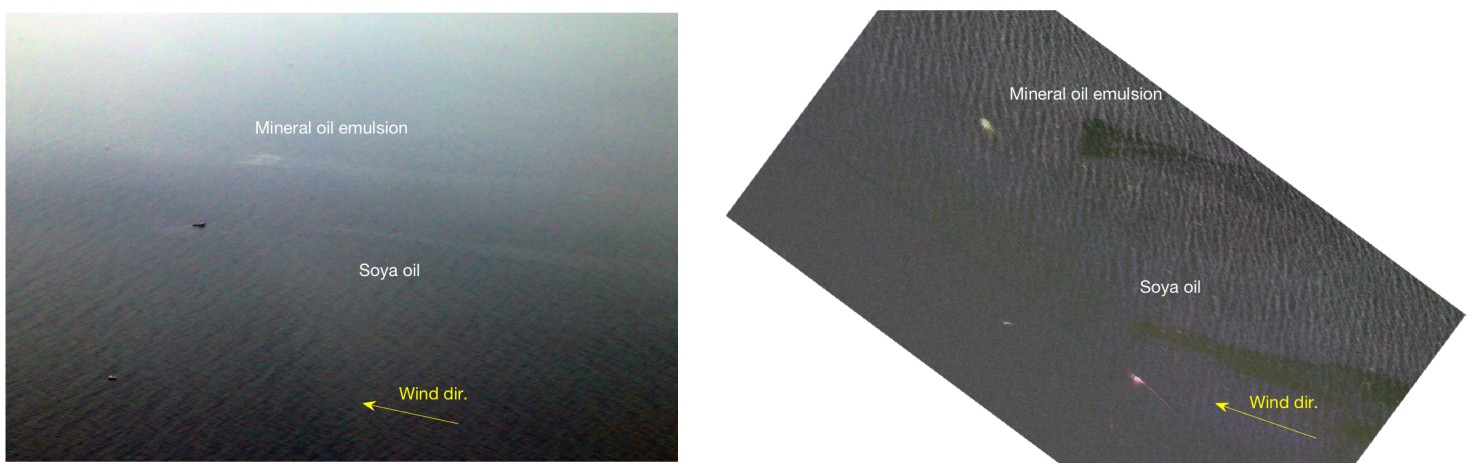

Fig. 10 Photograph taken from within the Dornier DO228-212 aircraft showing the two marine surface slicks, one of mineral oil emulsion and one of soybean oil, discharged during the NORSE 2019 oil-on-water campaign. In the image, the head of the slick of mineral oil emulsion is clearly visible with the tail of the slick also visible. Three ships can be seen in the left foreground, middle ground and background. Time of photograph: 10:46 UTC. The S-band SAR image to the right was acquired close to the time the photograph was taken. Both slicks are visible in this acquisition as well as the head and tail of the mineral oil emulsion slick. The three ships seen in the photograph are also visible are 3 bright points. Time of SAR acquisition 11:09 UTC. The wind direction is indicated in both images with the yellow arrow. Photograph: Ralph Horn. Images published with permission from NOFO.

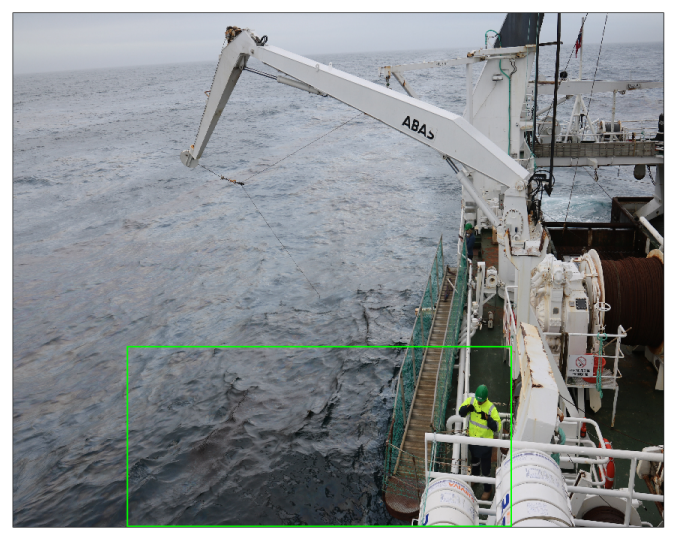

(a)

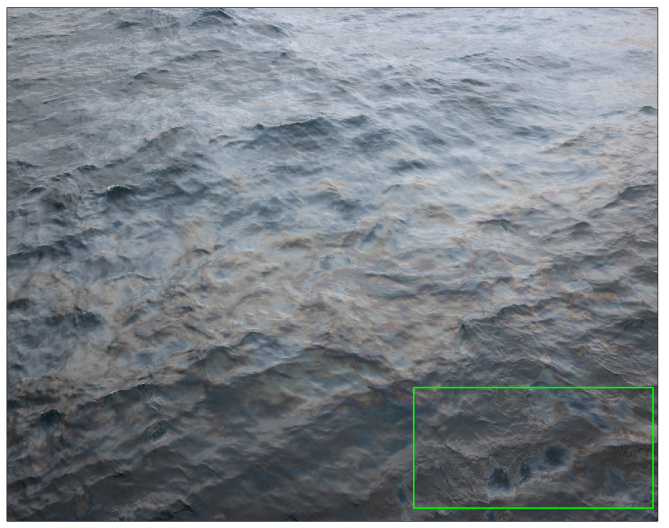

(c)

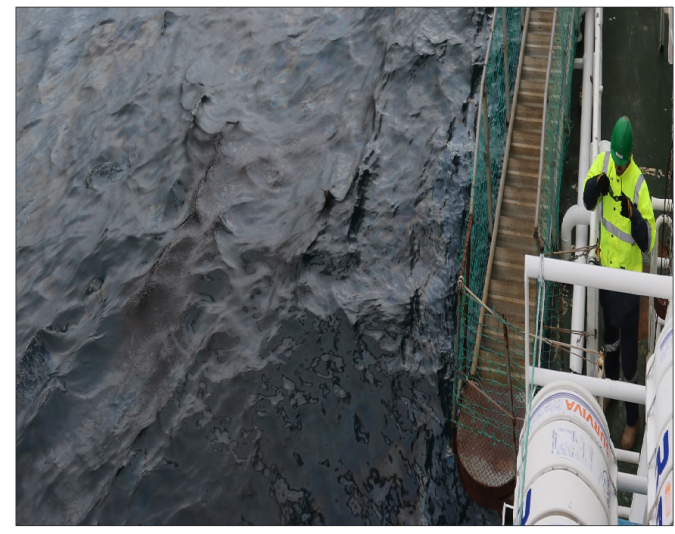

(b)

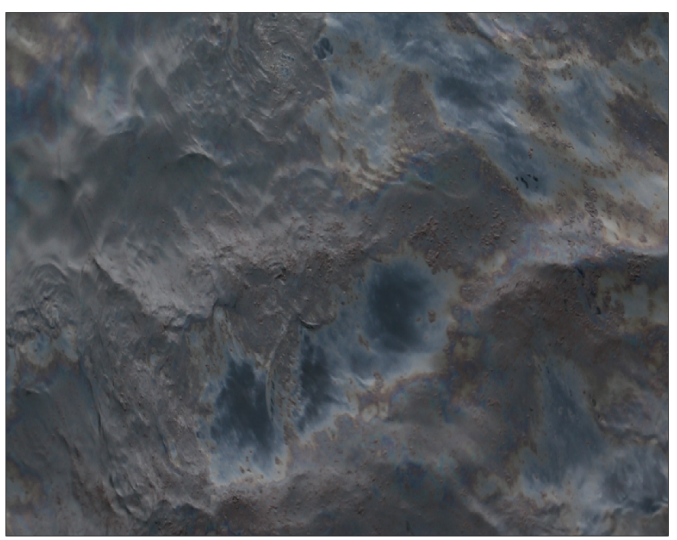

(d)

Fig. 11 (a) Photograph taken from the R/V Helmer Hanssen on 12 June 2019 at 10:30 UTC. A zone of thicker black oil can be seen in the middle foreground. (b) Area of image (a) shown in green square. Thicker zone of black oil is apparent. (c) Image of mineral oil emulsion slick taken from R/V Helmer Hanssen on 12 June 2019 at 10:30 UTC. (d) Area of image (c) bounded by green square. As can be seen the slick surface has a textured appearance. 


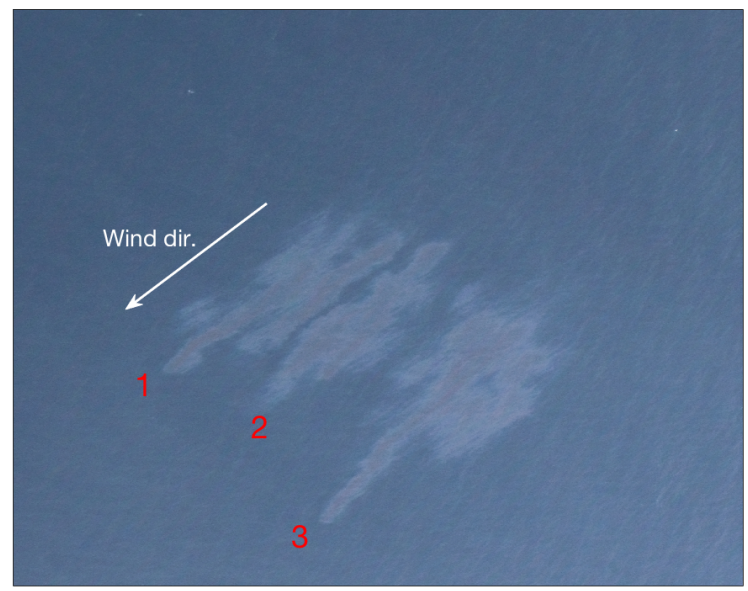

(a)

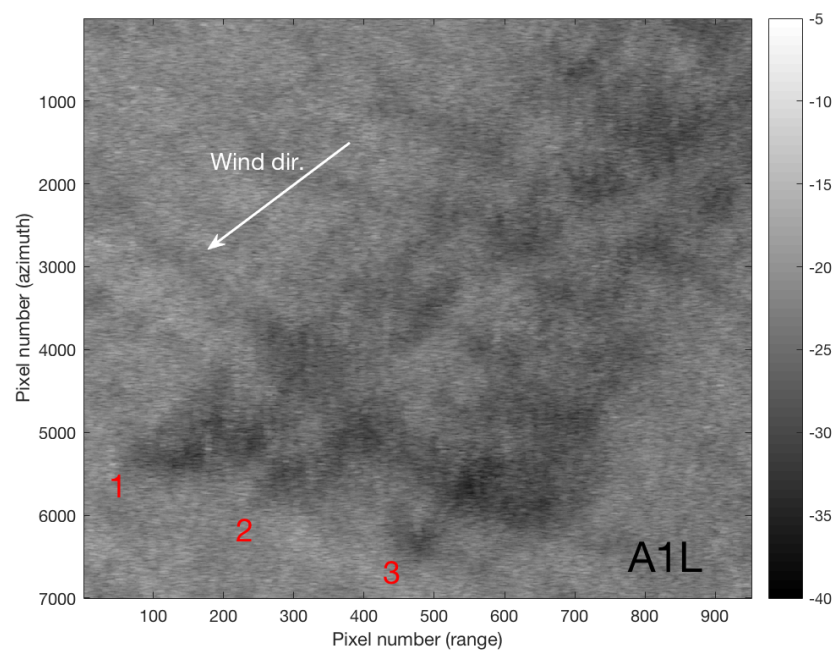

(c)

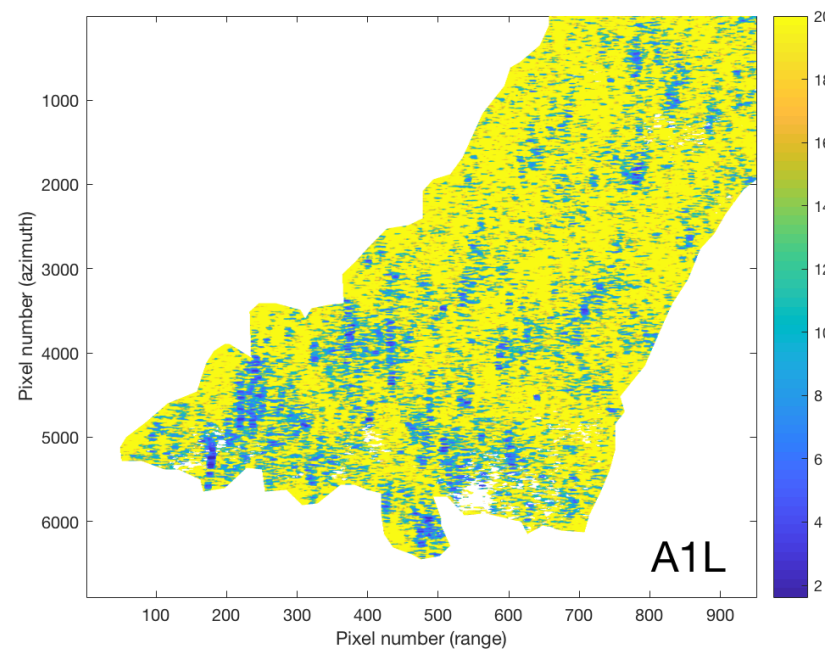

(e)

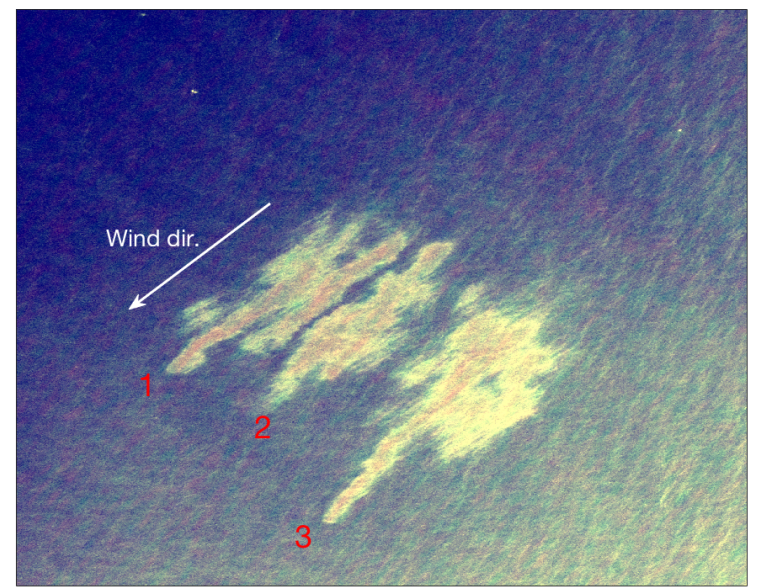

(b)

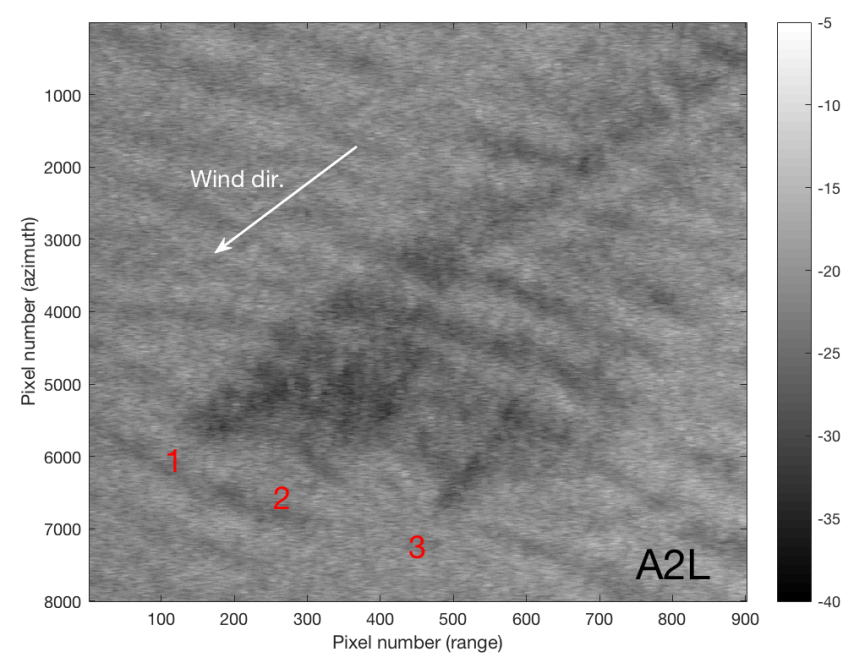

(d)

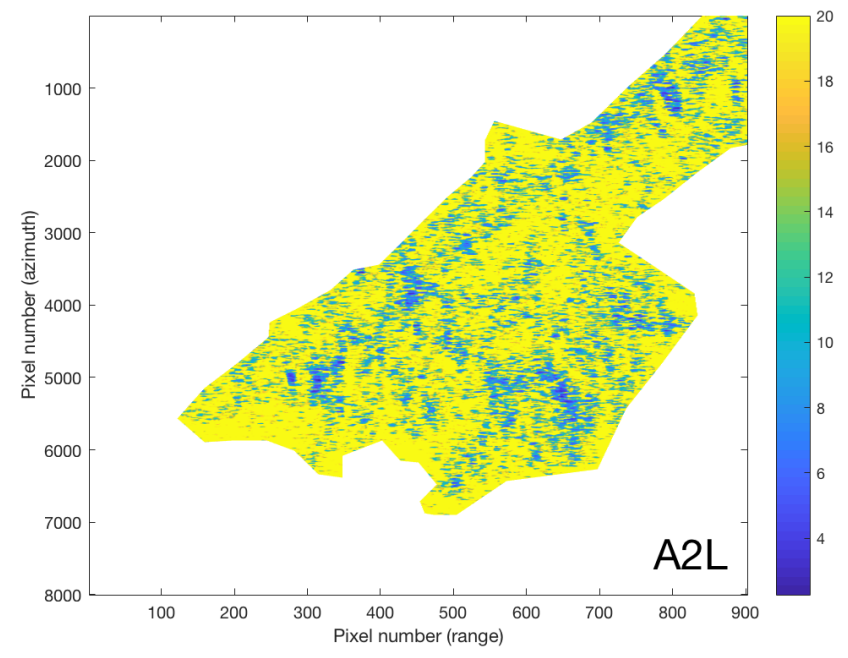

(f)

Fig. 12 (a) Photograph taken from the Dornier DO228-212 aircraft showing the mineral oil emulsion slick taken on 12 June 2019 at 11:30 UTC. (b) Same as in (a) but with processing performed on each color band for enhancement. (c), (d) L-band acquisition from A1 (A1L) and A2 (A2L) shown for reference. The tail of the slick is visible in both SAR acquisitions. SAR acquisitions taken on 12 June 2019 11:03 and 11:49 UTC, respectively. (e), (f) Inverted $|\varepsilon|$ values for A1L and A2L obtained from using method 2. Images reproduced from Fig. 8. 
the lifetime of a slick in which the PTSM is able to accurately determine dielectric values, interior to the slick, still needs to be determined. This is due to the effects of weathering i.e. emulsification and spreading, on the physical state of the slick, specifically water content and thickness, which can have direct impacts on the backscatter to the sensor.

This study provides an important contribution to the field as it indicates that in the absence of cross-polarization information, which leads to a system of equations that is underdetermined, accurate estimations for the dielectric properties of oil slick can still be derived.

Future work will explore the capability of the F-SAR instrument to determine accurate values for the dielectric properties of oil slick when some cross-polarization information from the surrounding ocean is available, but not from oil slick area. This would extend the viability of the PTSM to cases when satellite data with relatively low noise characteristics is available, or when the use of airborne SAR data is feasible.

The PTSM is given as

\section{Appendix A}

$$
\left\{\begin{array}{c}
\left\langle\sigma_{H H}^{0}\right\rangle_{/ a, b}=\frac{4}{\pi}\left[C_{0,0}^{H H}+\left[C_{2,0}^{H H}+2 \frac{\operatorname{Re}\left\{C_{0,0}^{H V}\right\}-C_{0,0}^{H H}}{\sin ^{2} v}+C_{0,2}^{H H}\right] s^{2}\right] \\
\left\langle\sigma_{V V}^{0}\right\rangle_{/ a, b}=\frac{4}{\pi}\left[C_{0,0}^{V V}+\left[C_{2,0}^{V V}+2 \frac{\operatorname{Re}\left\{C_{0,0}^{H V}\right\}-C_{0,0}^{V V}}{\sin ^{2} v}+C_{0,2}^{V V}\right] s^{2}\right] \\
\left\langle\sigma_{H V}^{0}\right\rangle_{/ a, b}=\frac{4}{\pi}\left[C_{0,0}^{H H}+C_{0,0}^{V V}-2 \operatorname{Re}\left\{C_{0,0}^{H V}\right\}\right] \frac{s^{2}}{\sin ^{2} v}
\end{array}\right.
$$

where $s^{2}$ is a measure of the large-scale roughness. The angle of incidence of the incoming radiation is indicated by $v$. The terms $C_{k, n-k}^{p q}$ are the series expansion coefficients of the function $\left(k \cos v_{l}\right)^{4} W F_{p} F_{q}^{*}$ and has the form

$$
C_{k, n-k}^{p q}=\left.\frac{1}{n !}\left(\begin{array}{l}
n \\
k
\end{array}\right) \frac{\partial^{n}\left(\left(k \cos v_{l}\right)^{4} W F_{p} F_{q}^{*}\right)}{\partial a^{k} \partial b^{n-k}}\right|_{a=b=0}
$$

The full expressions for $C_{0,0}^{p q}, C_{2,0}^{p q}$ and $C_{0,2}^{p q}$ were derived numerically. The subscripts and superscripts $p$ and $q$ indicate send and receive polarizations, respectively. The quantity $W$ is the power spectral density (PSD) of the small-scale roughness, with a roughness variance of $h^{2}$.The reader is again referred to [12] (and references therein) for an explicit treatment on this model. The Bragg coefficients, $F_{H}$ and $F_{V}$, are dependant on $|\varepsilon|$ and whose full expressions can be found in [12].

It should be noted however that implicit to the quantity $W$, there is another quantity, $H_{t}$, referred to as the Hurst coefficient. It was explicitly shown in [12] that the model is very weakly dependent on this quantity. Following [11], a value of 0.9 was chosen. An explanation for this choice can be found in that reference.

\section{Appendix B}

When using only the co- polarization ratio, we are left with one equation with two unknows, Quigley et. al. [11] proposed a method to estimate the $s$ parameter, from the surrounding ocean area, adjacent to oil slick before inversion is performed. The following provides a brief outline of the method proposed in that paper.

Fig. 13 (a) shows the PTSM co-polarization ratio plotted against $|\varepsilon|$ for a frequency of radiation equal to $5.4 \mathrm{GHz}$, a look angle of $40^{\circ}$ and $H_{t}$ equal to 0.9 with various values for $s$ indicated. This graph shows that the PTSM co-polarization ratio is highly sensitive to values of the roughness parameter $s$ with the PTSM being largely insensitive to $|\varepsilon|$ values over 30, approximately.

The high degree of sensitivity the model has to the values of $s$ suggests that this quantity needs to be estimated from localized patches of the ocean surface.

For this reason, the co-polarization ratio image was divided into square regions of size $100 \times 100$ pixels (in section IV.A we will also presented an analysis for the case when it is divided into square regions of size $50 \times 50$ pixels). The $s$ value for which the model curve approximates the mean of the co-polarization ratio scatter at a value of $|\varepsilon|$ equal to the value of sea water in that frequency band is taken as an estimate for $s$ in the $100 \times 100$ pixel sized area. The mean value is chosen as it was observed that the co-polarization ratio scatter has a Gaussian-like distribution over open ocean.

An example of the scatter of co-polarization ratio values within a single 100 pixel $\times 100$ pixel sized image portion of the ocean surface is shown in Fig. 13 (b) and is represented as the red, vertical scatter, and is plotted at a value of $|\varepsilon|$ equal to 75.43 $(\varepsilon=65.54-37.33 j)$ and where the blue $\mathrm{X}$ indicates the average of the scatter. This value was calculated using nominal inputs for the salinity and sea surface temperature of the North Sea in C-band. This value for $\varepsilon$ is consistent with [22] who determined the complex permittivity of sea water at $0^{\circ} \mathrm{C}$ and a salinity of $35 \mathrm{ppt}$ to be $64-40 \mathrm{j}$. The images shown in Fig. 13 were reproduced from [11] who used C-band Radarsat-2 data in their analysis and were included here as a reference for the methodology employed in this study.

Section IV illustrated that only S- and L-band acquisitions were suitable for analysis in this study. In order to determine the $|\varepsilon|$ value for open water during the NORSE 2019 exercise in those frequency bands, a nominal value for the salinity of the North Sea and in-situ measurements of sea surface temperature were used to calculate the dielectric properties of open water. The $|\varepsilon|$ for S-band was found to be 80.77 ( $\varepsilon=71.42-$ $37.74 j)$. and in L-band $95.8336(\varepsilon=74.59-60.17 j)$. These values are also consistent with [22].

\section{References}

[1] J. Coleman, Ed., Oil in the Sea III: Inputs, Fates and Effects. The National Academic Press, 2003.

[2]A. Carpenter, "European maritime safety agency CleanSeaNet activities in the North Sea. Oil pollution in the North Sea" in The Handbook of Environmental Chemistry, 41. Springer International Publishing Cham., pp.33-48. 

MONTH 20XX

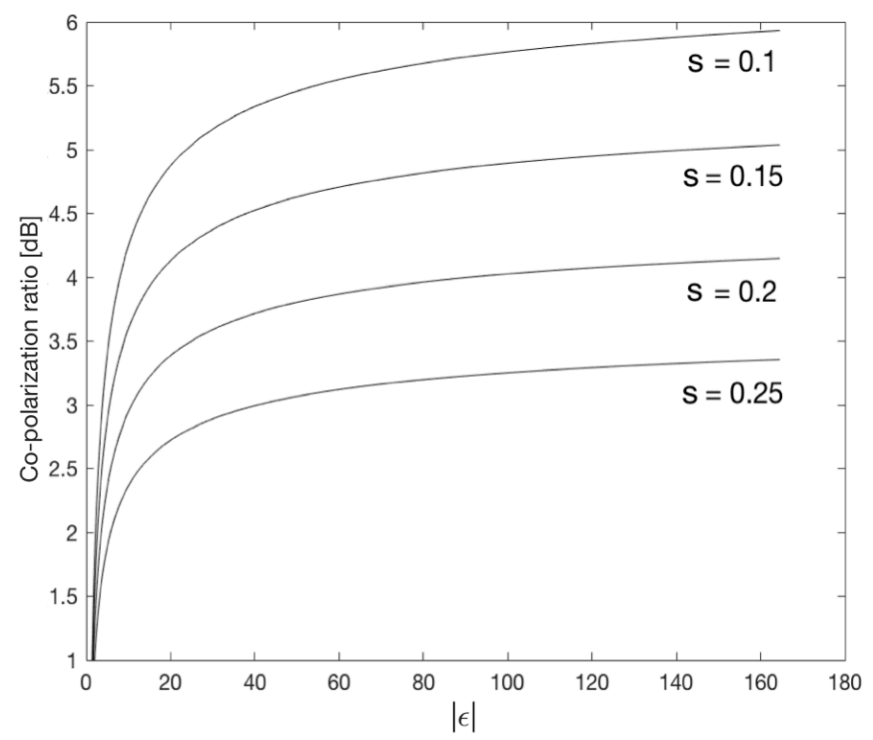

(a)

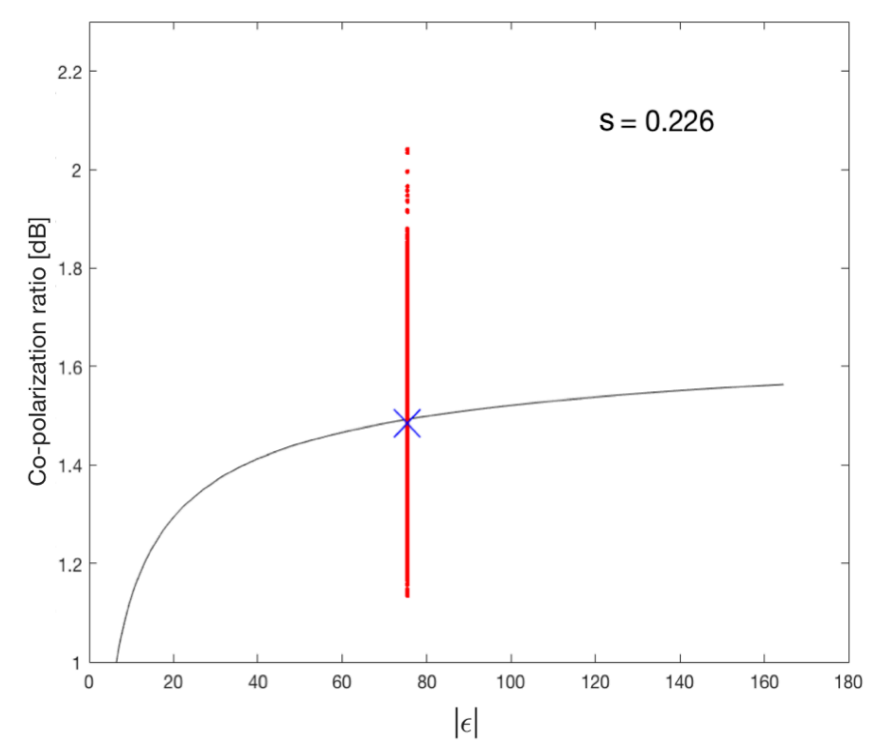

(b)

Fig. 13 (a) The PTSM co-polarization ratio computed for various values of $s$ with the same values for $H_{t}, v$ and radiation frequency as in Fig. 1. The models sensitivity to $s$ is apparent. (b) Method for estimating $s$. The red line is a scatter of points taken from a 100 pixel $\times 100$ pixel-sized portion of the co-pol image over clean ocean. The blue $\mathrm{X}$ is the mean of these pixel values. The $s$ value for which the PTSM curve coincides with the blue $X$ at the $|\varepsilon|$ value for water is assumed to be a valid estimation for $s$. Images reproduced from [11].

[3] M. J. Caruso, M. Migliaccio, J. T. Hargrove, J. T. GarciaPineda, and H. C. Graber, "Oil spills and slicks imaged by synthetic aperture radar," Oceanography, vol. 26, no. 2, pp. 112-123, 2013.

[4] B. Minchew, C. E. Jones, B. Holt, "Polarimetric analysis of backscatter from the Deepwater Horizon oil spill using Lband synthetic aperture radar", IEEE Trans. Geosci. Remote Sens., vol. 50, no. 10, pp. 3812-3830, Oct. 2012.
[5] B. Minchew, "Determining the mixing of oil and sea water using polarimetric synthetic aperture radar," Geophys. Res. Lett., vol. 39, 2012.

[6] S. Skrunes, C. Brekke, M.M. Espeseth, "Assessment of the RISAT-1 FRS-2 mode for oil spill observation" in IEEE IGARSS, Fort Worth, TX, USA, 2017, pp.1024-1027.

[7] ITOPF, "Aerial observations of marine oil spills," Inter. Tanker Owners Poll. Fed., London, UK, Tech. Info. Paper, no. 1,2011

[8] M. J. Collins, M. Denbina, B. Minchew, C. E. Jones, B. Holt, "On the use of simulated airborne compact polarimetric SAR for characterizing oil-water mixing of the Deepwater horizon oil spill", IEEE J. Sel. Topics Appl.

Earth Observ. Remote Sens., vol. 8, no. 3, pp. 1062-1077, Mar. 2015.

[9] Bonn Agreement Secretariat, "Bonn Agreement Aerial Operations Handbook", London, UK, Tech. Info. Paper, 2016.

Available: https://www.bonnagreement.org/site/assets/files/1081/aeria a_operations_handbook.pdf, Accessed on: Dec. 18, 2019.

[10] S. Angelliaume, O. Boisot, C.A. Guerin, "Dual-Polarized L-Band SAR Imagery for Temporal Monitoring of Marine Oil Slick Concentration," Remote Sens., vol. 10, no. 7, Jul. 2018.

[11] C. Quigley, C. Brekke, T. Eltoft, "Retrieval of Marine Surface Slick Dielectric Properties from Radarsat-2 Data via a Polarimetric Two-Scale Model", IEEE Trans. Geosci. Remote Sens., vol. 58, no. 7, pp. 5162-5178, July 2020.

[12] A. Iodice, A. Natale, D. Riccio, "Retrieval of soil surface parameters via a polarimetric two-scale model", IEEE Trans. Geosci. Remote Sens., vol. 49, no. 7, pp. 2531-2547, Jul. 2011.

[13] I. Hajnsek, E. Pottier, S. R. Cloude, "Inversion of surface parameters from polarimetric SAR", IEEE Trans. Geosci. Remote Sens., vol. 41, no. 4, pp. 727-744, Apr. 2003.

[14] C. Brekke, M.M. Espeseth, K. F. Dagestad, J. Röhrs, L. R. Hole and A. Reigber, "Integration of multi-sensor datasets and oil drift simulations - a free floating oil experiment in open ocean", JGR: Oceans, 2020 (unpublished manuscript).

[15]A.Reigber, M.Jager, M.Pinheiro, R.Scheiber, P.Prats, J.Fischer, R. Horn, A. Nottensteiner, "Performance of the Pband subsystem and the X-band interferometer of the FSAR airborne SAR instrument," in Geoscience and Remote Sensing Symposium (IGARSS), 2012 IEEE International, July 2012, pp. 5037-5040.

[16] G. R. Valenzuela, " Scattering of electromagnetic waves from a tilted slightly rough surface ", Radio Sci., vol. 3, no. 11, pp. 1057-1066, Jan. 1968.

[17] M. Espeseth, C. Brekke, C. Jones, B. Holt and A. Freeman, "The Impact of System Noise in Polarimetric SAR Imagery on Oil Spill Observations," IEEE Trans. Geosci. Remote Sens., vol. 58, no. 6, pp. 4194 - 4214, Jan. 2020.

[18] B. Slade, "Radarsat-2 Product Description," MacDonald, Dettwiler and Associates Ltd., Richmond, BC, USA, RNSP-52-1238, 2011.

[19] A. Freeman and S. Durden, "A three-component scattering model for polarimetric SAR data, "IEEE Trans. 

MONTH 20XX

Geosci. Remote Sens., vol. 36, no. 3,pp. 963-973, May 1998

[20] W. Alpers and H. Hühnerfuss, "Radar signatures of oil films floating on the sea surface and the Marangoni effect," J. Geophys. Res., vol. 93, no. C4, pp. 3642-3648, Apr. 1988.

[21] M. Fingas, "The challenges of remotely measuring oil slick thickness", Remote Sens., vol. 10, no. 2, pp. 319, Feb. 2018.

[22] T. Meissner, F. J. Wentz, "The complex dielectric constant of pure and sea water from microwave satellite observations", IEEE Trans. Geosci. Remote Sens., vol. 42, no. 9, pp. 1836-1849, Sep. 2004.

\section{Acknowledgments}

The authors wish to thank Veronique Miegebielle from Total E\&P. This work is funded by CIRFA through the RCN (grant no. 237906) and CIRFA partners. The authors would also sincerely like to thank NOFO for including our experiment in their exercise. In particular we would like to thank the crew from NOFO onboard RV HH and Kristin K. Husebye at NOFO HQ for her efforts in accommodating our numerous requests. We would like to thank DLR and the crew on board the F-SAR aircraft. In addition, we would also like to thank Oscar GarciaPineda from Water Mapping, LLC for allowing us to cite his oil thickness measurements.

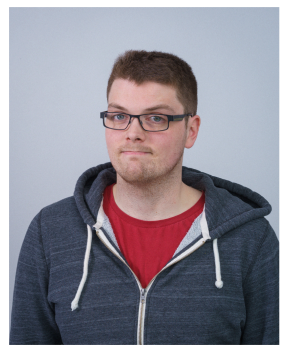

Cornelius Quigley received his Bachelor degree in Physics in June 2013 from the Department of Physics at the National University of Ireland, Cork. He received his Master's degree from the Department of Physics and Technology at the University of Tromsø - The Arctic University of Norway in June 2017. He started as a PhD student at the same department in September 2017, with the Center for Integrated Remote Sensing and Forecasting for Arctic Operations (CIRFA). His current research interests include remote sensing of ocean areas, specifically by polarimetric SAR and with a focus on marine oil pollution.

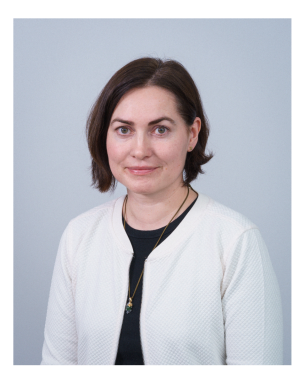

Camilla Brekke (M'12) received the Cand. Mag., Cand. Scient., and Ph.D. degrees from the Department of Informatics, University of Oslo, Oslo, Norway, in 1998, 2001, and 2008, respectively. She is currently the Vice-Dean Research with the Faculty of Science and Technology, the Deputy Centre Leader with the Centre for Integrated Remote Sensing and Forecasting for Arctic Operations and full Professor at Department of Physics and Technology, UiT The Arctic University of Norway, Troms $\varnothing$, Norway. Her current research interests include synthetic aperture radar and ocean color remote sensing for arctic and marine applications.

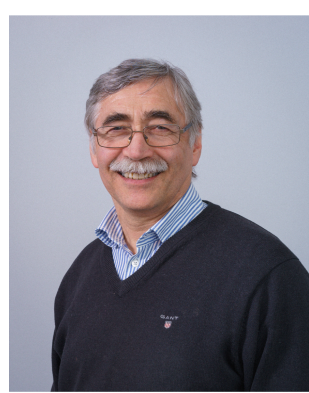

Torbjørn Eltoft (M'92) received the degrees of Cand. Real. (M.S.) and Dr. Scient. (Ph.D.) from University of Troms $\varnothing$ - The Arctic University of Norway, in 1981 and 1984, respectively. In 1988 he joined the Faculty of Science, University of Troms $\varnothing$ - The Arctic University of Norway, and is presently Professor in electrical engineering at the Department of Physics and Technology, where he also is head of the Earth Observation Laboratory and of the BARESS Remote Sensing School. He also holds a position as Adjunct Professor at Norut, Troms $ø$. His current research interests include multidimensional signal and image analysis with application in remote sensing, statistical models, neural networks, and machine learning. Dr. Eltoft has been associate editor of the journal Pattern Recognition for five years. He was awarded the year 2000 Outstanding Paper Award in Neural Networks by IEEE Neural Networks Council, and Honorable Mention for the 2003 Pattern Recognition Journal Best Paper Award. 